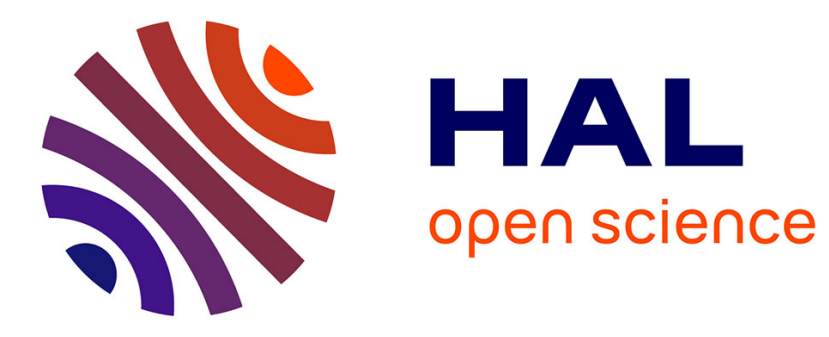

\title{
Borehole stability analysis in a thermoporoelastic dual-porosity medium
}

\author{
Rachel Gelet, Benjamin Loret, Nasser Khalili
}

\section{To cite this version:}

Rachel Gelet, Benjamin Loret, Nasser Khalili. Borehole stability analysis in a thermoporoelastic dual-porosity medium. International Journal of Rock Mechanics and Mining Science \& Geomechanics Abstracts, 2012, 50, pp.65-76. hal-00919454

\section{HAL Id: hal-00919454 https://hal.science/hal-00919454}

Submitted on 16 Dec 2013

HAL is a multi-disciplinary open access archive for the deposit and dissemination of scientific research documents, whether they are published or not. The documents may come from teaching and research institutions in France or abroad, or from public or private research centers.
L'archive ouverte pluridisciplinaire HAL, est destinée au dépôt et à la diffusion de documents scientifiques de niveau recherche, publiés ou non, émanant des établissements d'enseignement et de recherche français ou étrangers, des laboratoires publics ou privés. 


\title{
Borehole Stability Analysis in a Thermo-Poro-Elastic Dual Porosity Medium
}

\author{
Rachel Gelet ${ }^{\mathrm{a}, \mathrm{b}}$, Benjamin Loret $^{\mathrm{a}, *}$, Nasser Khalili ${ }^{\mathrm{b}}$ \\ ${ }^{a}$ Laboratoire Sols, Solides, Structures, B.P. 53X, 38041 Grenoble Cedex, France \\ ${ }^{b}$ School of Civil and Environmental Engineering, The University of New South Wales, Sydney 2052, Australia.
}

\begin{abstract}
The problem of diffusion and mass transfer in dual porous media is considered in a threephase framework. The solid phase is assumed to contain two distinct cavities filled with fluid. The porous mixture is composed of two overlapping media: the porous blocks and the fissure network. The fluid can transfer between the cavities due to fluid pressure difference. In addition, hydraulic and thermal diffusions take place through the mixture. A global understanding of mass transfer, diffusion and deformation is provided. The governing equations associated with these phenomena are presented for a mixture in thermal equilibrium. The finite element approximation of the governing equations is formulated and applied to the stability analysis of a vertical borehole. A parametric analysis is carried out to evaluate the influence of mass transfer on the pressure profiles of the fluids around the borehole. Permeable and a semipermeable boundary conditions are compared to predict the potential for failure of the wellbore under drained and partially undrained conditions.
\end{abstract}

Keywords: thermo-poro-elasticity, dual porosity, thermal equilibrium, diffusion dominated flow, mass transfer, borehole stability

\section{Introduction}

The influence of thermal loading on the behavior of fractured poro-elastic media is relevant to various applications, such as enhanced heavy-oil recovery by steam or hot water injection, thermal and hydraulic stimulations of tight reservoirs, management of nuclear waste disposal in a clay buffer, and geothermal hot dry rock energy extraction. In all these applications, a borehole or a tunnel is required and the stability analysis forms a crucial part of the design [1].

Currently borehole stability analyses under thermal loading are scarce and focus mainly on reservoirs represented by saturated rocks with a single porosity $[2,3,4,5,6,7,8,9,10,11,12]$. A key factor influencing wellbore stability is the change of pore pressure on the lining of the wellbore due to mud penetration $[5,6,7]$. Failure typically occurs when the pore pressure reduces the effective confining pressure below a threshold value $[8,9]$.

Closed form solutions for thermally induced fluid flow around a borehole in low permeability media, where heat transfer is dominated by conduction, have been presented by a number of investigators $[2,3,4,6]$. McTigue $[2,3]$ provided analytical results for the thermoelastic response

\footnotetext{
*Principal corresponding author

Email addresses: rachel.gelet@gmail.com (Rachel Gelet), benjamin.loret@hmg.inpg.fr (Benjamin Loret), n.khalili@unsw.edu.au (Nasser Khalili)
} 
of saturated porous rocks with a single porosity, highlighting the importance of the thermal to hydraulic diffusivity ratio and the competition between the heat and the fluid flows. Wang and Papamichos [4] examined the thermally induced pore fluid pressure around a pumping well for both cold and warm injection processes, underlying the importance of the thermal coupling to accurately estimate the induced fluid flow rate in low permeability media. Chen and Ewy [6] investigated the thermoporoelastic effect on wellbore stability and analyzed the collapse failure index in the region near the wellbore. Notable contributions have also been made by Abousleiman and Ekbote [10], Wang and Dusseault [11] and Pao et al. [12] on the effects of anisotropy, conductive versus convective heat flow, and multi-phase flow on borehole instability.

Based on Biot's theory of mixture [13], thermo-hydro-mechanical models have been extended to account for the dual porosity concept introduced by Barenblatt et al. [14]. However, the existing literature focuses on consolidation [15] or on the dominance of convection over conduction phenomena [16, 17]. Nair et al. [17, 18] presented results on the sensitivity of the thermoelastic response in dual porosity media to fracture spacing, but based on several restrictions. In particular, the deformation field in their dual porosity model was formulated by assigning each cavity system with its own effective stress, deformation and overall compliance, in contradiction of the principle of effective stress where a single stress entity is defined for the entire solid skeleton [19, 21, 22, 20]. Furthermore, in their approach, to define the effective stress parameters, the deformation fields of pore and fracture systems are assumed to work in series which may not be applicable in real rocks.

The paper presents a fully coupled finite element formulation for a thermo-poro-elastic dual porous medium under non-isothermal conditions. The fractured porous medium is described as a porous mixture composed of two overlapping continua: the porous blocks and the fissure network. The solid phase has a special role as it provides the matrix skeleton and encloses the fluid phases. The theoretical model of the three-phase mixture is built by postulating constitutive equations for the three phases and by enforcing the balances of mass, momentum and energy. A summary of the governing differential equations is provided in Section 2. The formulation presented is specified for a local thermal equilibrium between the phases, saturated soils and for diffusion dominated fluxes $[23,16]$. The weak form of the governing equations and the time-integration procedure to solve the coupled equations through a finite element method are detailed in Section 3. The primary variables are the displacements, the pore fluid pressure, the pressure of the fluid in the fissures and the temperature of the mixture. The resulting system of equations is used to address the failure potential of a vertical borehole subjected to both pressure and temperature gradients (Section 4). Simulations of heavy-oil recovery through thermal stimulation demonstrate the influences of the dual porosity approach and of the boundary conditions on the borehole stability (Section 5). In addition to the stability issue, the study focuses on mass transfer between the pore matrix and the fissure network; and on the contrast in diffusivity ratios between the two cavities.

Notation: Vector and matrix quantities are identified by boldface letters, for example $\boldsymbol{\sigma}$ is the total stress. I is the identity matrix. tr, $\boldsymbol{\nabla}(\cdot)$ and div denote respectively the trace, the gradient and the divergence operators.

\section{Governing equations}

Within a dual porosity conceptual framework (Khalili and Valliappan [19] and Khalili and Selvadurai [16]), differential equations describing the deformation, hydraulic and heat flows, and mass transfer through deformable fissured porous media may be expressed as (the convention 
of summation over the repeated mute index $i$ is used),

$$
\begin{aligned}
& \mu \frac{\partial^{2} u_{j}}{\partial x_{i} \partial x_{i}}+(\lambda+\mu) \frac{\partial^{2} u_{i}}{\partial x_{i} \partial x_{j}}-\xi_{p} \frac{\partial p_{p}}{\partial x_{j}}-\xi_{f} \frac{\partial p_{f}}{\partial x_{j}}-\frac{c_{T}}{c} \frac{\partial T}{\partial x_{j}}+F_{j}=0 \\
& \frac{\partial}{\partial x_{i}}\left(\frac{k_{p}}{\mu_{p}} \frac{\partial p_{p}}{\partial x_{i}}\right)=a_{p p} \frac{\partial p_{p}}{\partial t}+a_{p f} \frac{\partial p_{f}}{\partial t}+\xi_{p} \frac{\partial^{2} u_{i}}{\partial t \partial x_{i}}+a_{p T} \frac{\partial T}{\partial t}+\Gamma \\
& \frac{\partial}{\partial x_{i}}\left(\frac{k_{f}}{\mu_{f}} \frac{\partial p_{f}}{\partial x_{i}}\right)=a_{f f} \frac{\partial p_{f}}{\partial t}+a_{p f} \frac{\partial p_{p}}{\partial t}+\xi_{f} \frac{\partial^{2} u_{i}}{\partial t \partial x_{i}}+a_{f T} \frac{\partial T}{\partial t}-\Gamma \\
& \frac{\partial}{\partial x_{i}}\left(\Lambda \frac{\partial T}{\partial x_{i}}\right)=T\left(a_{p T} \frac{\partial p_{p}}{\partial t}+a_{f T} \frac{\partial p_{f}}{\partial t}+\frac{c_{T}}{c} \frac{\partial^{2} u_{i}}{\partial t \partial x_{i}}\right)+a_{T T} \frac{\partial T}{\partial t}
\end{aligned}
$$

in which,

$$
\begin{array}{llll}
\xi_{p} & =\left(c_{p}-c_{s}\right) / c, & \xi_{f} & =1-c_{p} / c \\
a_{p f} & =-\left(\xi_{f}-n_{f}\right)\left(c_{p}-c_{s}\right), & a_{p p} & =n_{p} c_{H, p}+\left(\xi_{p}-n_{p}\right) c_{s}-a_{p f} \\
a_{f f} & =n_{f} c_{H, f}+\left(\xi_{f}-n_{f}\right) c_{s}-a_{p f}, & & a_{p T}=\left(n_{p}-\xi_{p}\right) c_{T}-n_{p} c_{T, p} \\
a_{f T} & =\left(n_{f}-\xi_{f}\right) c_{T}-n_{f} c_{T, f}, & & a_{T T}=\rho C_{p}, \\
\Gamma & =\eta\left(p_{p}-p_{f}\right), & \eta & =\bar{\alpha} k_{p} / \mu_{p}, \\
\bar{\alpha} & =4 n(n+2) / l^{2} \quad \text { with } n=1,2,3, & &
\end{array}
$$

where $\mathbf{u}=\left(u_{j}\right)$ is the displacement of the solid phase, $p_{p}$ is the pressure of the pore fluid, $p_{f}$ is the pressure of the fissure fluid and the temperature of the mixture, which is in thermal equilibrium, is denoted $T$. The subscripts $s, p, f$ refer to the solid skeleton, the pore fluid and the fissure fluid, respectively.

Equation $(1)_{1}$ uses the Lamé constants $\lambda$ and $\mu$ of the drained solid, the body force vector $F_{i}$, the compressibility of the mixture $c$, the compressibility of the porous blocks $c_{p}$, the compressibility of the solid grains $c_{s}$, and the volumetric thermal expansion coefficient of the solid phase $c_{T}$. Equations $(1)_{2,3}$ require for each fluid $k=p, f$ the macroscopic porosity $n_{k}$, the macroscopic intrinsic permeability $k_{k}$, and the dynamic viscosity $\mu_{k}$. The hydraulic compressibility $c_{H, k}$ and the thermal compressibility $c_{T, k}$ are defined in eqn (11) from the intrinsic density $\rho_{k}$ of the fluid $k$. A linear mass transfer function $\Gamma=\eta\left(p_{p}-p_{f}\right)$ defining the exchange of fluid between the porous blocks and the fissure network is adopted [14, 24]. $\eta$ is the leakage parameter defined by Warren and Root [24] through the factor $\bar{\alpha}$ as a function of the average fissure spacing $l$ and the number $n$ of normal sets of fissures. While the linear transfer function is easily amenable to computational implementation and consistent with a thermodynamic analysis, leading to positive dissipation, it is also known to be inaccurate at early times. The non linear Vermeulen scheme has been adopted by Zimmerman et al. [32] in the analysis of fractured geothermal reservoirs where, at each point of the fracture continuum, a porous block of spherical shape is attached: the fluid diffuses in the block and the net flow through its boundary is viewed as a source/sink term for the fracture continuum. Lu and Connell [33] have devised a one-dimensional semi-analytical scheme that provides the time course of the transferred mass in a gas reservoir. At early times, while the rate of mass transfer in their model tends to vanish, it tends to a constant for the linear transfer scheme and to infinity for the Vermeulen scheme. Correspondingly, the mass transferred depends linearly on time in the linear transfer scheme, but on the square root of time in the schemes of Vermeulen and $\mathrm{Lu}$ and Connell, albeit with distinct scaling factors. A simple, accurate while computationally efficient, model of transfer that avoids delving with a convolution product, is yet to come. 
Equation $(1)_{4}$ involves the overall heat capacity at constant strain and fluid pressure $C_{p}$, the overall density $\rho$, and the overall thermal conductivity $\Lambda$.

Equations (1) are direct consequences of the field equations (balance of momentum for the mixture, balance of mass for each fluid phase, balance of energy for the mixture),

$$
\begin{aligned}
-\operatorname{div} \boldsymbol{\sigma} & =\mathbf{F}, \\
-\operatorname{div} \mathbf{J}_{k} & =n_{k} \frac{1}{\rho_{k}} \frac{\mathrm{d}^{k} \rho_{k}}{d t}+\frac{1}{V} \frac{\mathrm{d} V_{k}}{\mathrm{~d} t}-\frac{\hat{\rho}_{t r}^{k}}{\rho_{k}}, \quad k=p, f, \\
-\operatorname{div} \mathbf{q} & =T \frac{\mathrm{d} s}{\mathrm{~d} t},
\end{aligned}
$$

where $\boldsymbol{\sigma}$ is the total stress, $\mathbf{F}$ is the body force vector, $\mathbf{J}_{k}$ is the flux of the fluid $k$ and $\mathbf{q}$ is the heat flux of the mixture. The term $\hat{\rho}_{t r}^{k}$ represents the mass supply to the cavity $k$ by the other cavity.

The initial configuration, which is taken as a reference, represents a state in equilibrium in which stress, strain, pressures and temperature can be non-zero. Departure from this reference state is denoted $\Delta($.$) . The shear behavior is accounted for by the shear modulus \mu$ of the (drained) solid skeleton eqn (40). The fluid does not react to shear stresses. The associated relationship links the elastic strain $\boldsymbol{\epsilon}^{e l}$ to the effective stress $\boldsymbol{\sigma}^{\prime}$,

$$
\operatorname{tr} \boldsymbol{\epsilon}^{e l}=c \frac{\operatorname{tr} \boldsymbol{\sigma}^{\prime}}{3}, \quad \operatorname{dev} \boldsymbol{\epsilon}^{e l}=\frac{\operatorname{dev} \boldsymbol{\sigma}^{\prime}}{2 \mu}
$$

in which the deviatoric parts of the elastic strain and of the effective stress are denoted dev $\epsilon^{e l}$ and $\operatorname{dev} \boldsymbol{\sigma}^{\prime}$, respectively. The thermo-mechanical constitutive matrix relates the isotropic part of the total stress tr $\boldsymbol{\sigma} / 3$, the volume variations of the fluids $\Delta V_{p} / V$ and $\Delta V_{f} / V$, and the entropy variation of the solid $\Delta s_{s}$ to the isotropic part of the total strain $\operatorname{tr} \epsilon$, the fluid pressures $p_{p}, p_{f}$ and the temperature change $\Delta T=T-T^{0}$ by,

$$
\left[\begin{array}{c}
-\operatorname{tr} \boldsymbol{\sigma} / 3 \\
\Delta V_{p} / V \\
\Delta V_{f} / V \\
\Delta s_{s}
\end{array}\right]=\left[\begin{array}{cccc}
-1 / c & \xi_{p} & \xi_{f} & c_{T} / c \\
\xi_{p} & a_{p p}-n_{p} c_{H, p} & a_{p f} & \left(n_{p}-\xi_{p}\right) c_{T} \\
\xi_{f} & a_{p f} & a_{f f}-n_{f} c_{H, f} & \left(n_{f}-\xi_{f}\right) c_{T} \\
c_{T} / c & \left(n_{p}-\xi_{p}\right) c_{T} & \left(n_{f}-\xi_{f}\right) c_{T} & \rho^{s} C_{p, s} / T
\end{array}\right]\left[\begin{array}{c}
\operatorname{tr} \boldsymbol{\epsilon} \\
p_{p} \\
p_{f} \\
\Delta T
\end{array}\right]
$$

in which $\rho^{s}$ is the apparent density of the solid equal to $n_{s} \rho_{s}, s_{s}$ is the apparent entropy of the solid per unit volume $\left[\mathrm{kg} / \mathrm{m} / \mathrm{s}^{2}\right]$ and $C_{p, s}$ is the intrinsic heat capacity of the solid, at constant strain and fluid pressure $[\mathrm{J} / \mathrm{kg} . \mathrm{K}] . \xi_{p}$ and $\xi_{f}$ are the effective stress parameters, which define the hydro-mechanical coupling and $a_{p f}$ is the coupling term ensuring compatibility of deformation between the two pore systems [25]. The apparent entropy variation of the generic fluid $k$ is expressed separately as,

$$
\Delta s_{k}=-n_{k} c_{T, k} p_{k}+\rho^{k} \frac{C_{p, k}}{T} \Delta T, \quad k=p, f .
$$

By summing the contributions of the three phases, the entropy variation for the mixture introduces the heat capacity of the porous medium $\rho C_{p}=\rho^{s} C_{p, s}+\rho^{p} C_{p, p}+\rho^{f} C_{p, f}$,

$$
\Delta s=\frac{c_{T}}{c} \operatorname{tr} \epsilon+a_{p T} p_{p}+a_{f T} p_{f}+\frac{\rho C_{p}}{T} \Delta T .
$$


The density of the thermo-barotropic fluid $k$ varies with its pressure and temperature,

$$
\frac{1}{\rho_{k}} \frac{\mathrm{d}^{k} \rho_{k}}{\mathrm{~d} t}=c_{H, k} \frac{\mathrm{d}^{k} p_{k}}{\mathrm{~d} t}-c_{T, k} \frac{\mathrm{d}^{k} T}{\mathrm{~d} t}, \quad k=p, f
$$

in which the compressibility $c_{H, k}$ and the thermal expansion $c_{T, k}$ are defined as,

$$
c_{H, k}=\left.\frac{1}{\rho_{k}} \frac{\mathrm{d}^{k} \rho_{k}}{\mathrm{~d} p_{k}}\right|_{T_{k}}, \quad c_{T, k}=-\left.\frac{1}{\rho_{k}} \frac{\mathrm{d}^{k} \rho_{k}}{\mathrm{~d} T}\right|_{p_{k}}, \quad k=p, f .
$$

The diffusion constitutive relations are described by uncoupled Darcy's law and Fourier's law,

$$
\mathbf{J}_{k}=-\frac{k_{k}}{\mu_{k}} \nabla p_{k}, \quad k=p, f ; \quad \mathbf{q}=-\Lambda \nabla T,
$$

while the mass transfer uses Barenblatt's quasi-steady relationship [14],

$$
\hat{\rho}_{t r}^{k}=(-1)^{\alpha} \rho_{0} \eta\left(p_{p}-p_{f}\right),
$$

in which $\eta$ is the leakage parameter, which controls the mass transfer due to the fluid pressure difference between the two cavities, and $\alpha=1$ for $k=p, \alpha=2$ for $k=f$.

This thermo-hydro-mechanical model derives from an effective stress concept, eqn $(7)_{1}$,

$$
\frac{\operatorname{tr} \boldsymbol{\sigma}}{3}+\xi_{p} p_{p}+\xi_{f} p_{f}=\frac{\operatorname{tr} \boldsymbol{\epsilon}}{c}-\frac{c_{T}}{c} \Delta T
$$

and accounts for hydraulic fluxes within the two cavities, overall heat flux and fluid exchanges between the two cavities. This model neglects convective effects, the gravity force and the coupled diffusive terms between the hydraulic fluxes and the heat flux. Additional information on the parameters of the model can be found in previous works [19], [16] and [26].

\section{Finite element formulation}

A mixed finite element formulation is developed in which the primary unknowns are the displacements $\mathbf{u}$, the pore pressure $p_{p}$, the pressure of the fissure fluid $p_{f}$ and the temperature of the porous medium $T$. To compact the finite element formulation, it is instrumental to introduce the functions $f_{p}, f_{f}$ and $f_{T}$ defined as,

$$
\begin{aligned}
& f_{p}=a_{p p} \dot{p}_{p}+a_{p f} \dot{p}_{f}+a_{p T} \dot{T}+\xi_{p} \operatorname{div} \dot{\mathbf{u}}+\eta\left(p_{p}-p_{f}\right), \\
& f_{f}=a_{f p} \dot{p}_{p}+a_{f f} \dot{p}_{f}+a_{f T} \dot{T}+\xi_{f} \operatorname{div} \dot{\mathbf{u}}-\eta\left(p_{p}-p_{f}\right), \\
& f_{T}=T a_{p T} \dot{p}_{p}+T a_{f T} \dot{p}_{f}+a_{T T} \dot{T}+T \frac{c_{T}}{c} \operatorname{div} \dot{\mathbf{u}},
\end{aligned}
$$

in which the super-imposed dot indicates a partial time derivative.

\subsection{The semi-discrete equations}

Multiplying the field equations by the virtual fields $\delta \mathbf{u}, \delta p$ and $\delta T$ and integrating by parts over the body $V$ provides the weak form of the problem,

$$
\begin{aligned}
-\int_{V} \boldsymbol{\nabla}(\delta \mathbf{u}): \boldsymbol{\sigma} d V+\int_{V} \delta \mathbf{u} \cdot \mathbf{F} d V & =-\int_{\partial V} \delta \mathbf{u} \cdot \boldsymbol{\sigma} \cdot \hat{\mathbf{n}} d S, \\
-\int_{V} \boldsymbol{\nabla}(\delta p) \cdot \mathbf{J}_{k} d V+\int_{V} \delta p f_{k} d V & =-\int_{\partial V} \delta p \mathbf{J}_{k} \cdot \hat{\mathbf{n}} d S, \quad \text { for } \quad k=p, f ; \\
-\int_{V} \boldsymbol{\nabla}(\delta T) \cdot \mathbf{q} d V+\int_{V} \delta T f_{T} d V & =-\int_{\partial V} \delta T \mathbf{q} \cdot \hat{\mathbf{n}} d S,
\end{aligned}
$$


where $\hat{\mathbf{n}}$ is the unit outward normal to the boundary $\partial V$. A generalized Galerkin procedure is adopted and the same interpolation functions are used for the primary unknowns and for the variations. The primary unknowns are interpolated, within the generic element $e$, in terms of nodal values through the interpolation functions $\mathbf{N}_{\mathbf{u}}, \mathbf{N}_{\mathbf{p}}, \mathbf{N}_{\mathbf{T}}$, respectively,

$$
\mathbf{u}=\mathbf{N}_{\mathbf{u}} \mathbf{u}^{e} ; \quad p_{k}=\mathbf{N}_{\mathbf{p}} \mathbf{p}_{\mathbf{k}}^{e}, \quad \text { for } \quad k=p, f ; \quad T=\mathbf{N}_{\mathbf{T}} \mathbf{T}^{e} .
$$

The surface loading contributions of a generic element $e$ are gathered into the vector $\mathbb{F}_{e}^{\text {surf }}$ and the internal thermo-poro-elastic contributions into the vector $\mathbb{F}_{e}^{\text {int }}$. The element contributions to the force vectors (16) are equal to $\mathbb{F}_{e}^{\text {int }}-\mathbb{F}_{e}^{\text {surf }}$,

$$
\left[\begin{array}{c}
\int_{V^{e}}\left(\mathbf{B}_{\mathbf{u}}\right)^{\mathrm{T}} \boldsymbol{\sigma}-\left(\mathbf{N}_{\mathbf{u}}\right)^{\mathrm{T}} \mathbf{F} d V^{e} \\
\int_{V^{e}}\left(\boldsymbol{\nabla} \mathbf{N}_{\mathbf{p}}\right)^{\mathrm{T}} \mathbf{J}_{p}-\left(\mathbf{N}_{\mathbf{p}}\right)^{\mathrm{T}} f_{p} d V^{e} \\
\int_{V^{e}}\left(\boldsymbol{\nabla} \mathbf{N}_{\mathbf{p}}\right)^{\mathrm{T}} \mathbf{J}_{f}-\left(\mathbf{N}_{\mathbf{p}}\right)^{\mathrm{T}} f_{f} d V^{e} \\
\int_{V^{e}}\left(\nabla \mathbf{N}_{\mathbf{T}}\right)^{\mathrm{T}} \mathbf{q}-\left(\mathbf{N}_{\mathbf{T}}\right)^{\mathrm{T}} f_{T} d V^{e}
\end{array}\right]-\left[\begin{array}{c}
\int_{\partial V^{e}}\left(\mathbf{N}_{\mathbf{u}}\right)^{\mathrm{T}} \boldsymbol{\sigma} \cdot \hat{\mathbf{n}} d S^{e} \\
\int_{\partial V^{e}}\left(\mathbf{N}_{\mathbf{p}}\right)^{\mathrm{T}} \mathbf{J}_{p} \cdot \hat{\mathbf{n}} d S^{e} \\
\int_{\partial V^{e}}\left(\mathbf{N}_{\mathbf{p}}\right)^{\mathrm{T}} \mathbf{J}_{f} \cdot \hat{\mathbf{n}} d S^{e} \\
\int_{\partial V^{e}}\left(\mathbf{N}_{\mathbf{T}}\right)^{\mathrm{T}} \mathbf{q} \cdot \hat{\mathbf{n}} d S^{e}
\end{array}\right]
$$

where $\mathbf{B}_{\mathbf{u}}$ is the standard strain-displacement matrix, namely $\boldsymbol{\epsilon}=\mathbf{B}_{\mathbf{u}} \mathbf{u}^{e}$. Inserting the total stress $(7)_{1}$ and the hydraulic and thermal fluxes (12) in (18) yields a non-linear system of equations including

- an element contribution to the balance of momentum of the mixture,

$$
\mathbf{E}_{\mathbf{u u}}^{e} \mathbf{u}^{e}-\mathbf{C}_{\mathbf{u p}}^{e}\left(\xi_{p} \mathbf{p}_{\mathbf{p}}^{e}+\xi_{f} \mathbf{p}_{\mathbf{f}}^{e}\right)-\frac{c_{T}}{c} \mathbf{C}_{\mathbf{u} \mathbf{T}}^{e} \mathbf{T}^{e}-\mathbf{R}_{\mathbf{u}}^{e},
$$

- an element contribution to the balance of mass of the pore fluid,

$$
\begin{aligned}
& -\left[\mathbf{J}_{\mathbf{k}_{\mathbf{p}}}^{e}+\eta \mathbf{M}_{\mathbf{p} \mathbf{p}}^{e}\right] \mathbf{p}_{\mathbf{p}}{ }^{e}+\eta \mathbf{M}_{\mathbf{p} \mathbf{p}}^{e} \mathbf{p}_{\mathbf{f}}{ }^{e} \\
& -\mathbf{M}_{\mathbf{p} \mathbf{p}}^{e}\left(a_{p p} \dot{\mathbf{p}}_{\mathbf{p}}^{e}+a_{p f} \dot{\mathbf{p}}_{\mathbf{f}}^{e}\right)-\mathbf{M}_{\mathbf{p} \mathbf{T}}^{e} a_{p T} \dot{\mathbf{T}}^{e}-\xi_{p} \mathbf{C}_{\mathbf{p u}}^{e} \dot{\mathbf{u}}^{e}-\mathbf{R}_{\mathbf{p}_{\mathbf{p}}}^{e},
\end{aligned}
$$

- an element contribution to the balance of mass of the fissure fluid,

$$
\begin{aligned}
& -\left[\mathbf{J}_{\mathbf{k}_{\mathbf{f}}}^{e}+\eta \mathbf{M}_{\mathbf{p} \mathbf{p}}^{e}\right] \mathbf{p}_{\mathbf{f}}^{e}+\eta \mathbf{M}_{\mathbf{p} \mathbf{p}}^{e} \mathbf{p}_{\mathbf{p}}^{e} \\
& -\mathbf{M}_{\mathbf{p} \mathbf{p}}^{e}\left(a_{f f} \dot{\mathbf{p}}_{\mathbf{f}}^{e}+a_{p f} \dot{\mathbf{p}}_{\mathbf{p}}^{e}\right)-\mathbf{M}_{\mathbf{p} \mathbf{T}}^{e} a_{f T} \dot{\mathbf{T}}^{e}-\xi_{f} \mathbf{C}_{\mathbf{p u}}^{e} \dot{\mathbf{u}}^{e}-\mathbf{R}_{\mathbf{p}_{\mathbf{f}}}^{e},
\end{aligned}
$$

- an element contribution to the balance of energy of the mixture,

$$
-\mathbf{Q}_{\boldsymbol{\Lambda}}^{e} \mathbf{T}^{e}-\mathbf{M}_{\mathbf{p} \mathbf{T}}^{e \mathrm{~T}} T\left(a_{p T} \dot{\mathbf{p}}_{\mathbf{p}}^{e}+a_{f T} \dot{\mathbf{p}}_{\mathbf{f}}^{e}\right)-a_{T T} \mathbf{M}_{\mathbf{T} \mathbf{T}}^{e} \dot{\mathbf{T}}^{e}-T \frac{c_{T}}{c} \mathbf{C}_{\mathbf{T u}}^{e} \dot{\mathbf{u}}^{e}-\mathbf{R}_{\mathbf{T}}^{e}
$$

Details of the element force vectors and matrices are provided in Appendix A. The contribution of the element $e$ to the global set of equations may be cast in a matrix format,

$$
\mathbb{K}^{e} \mathbb{X}^{e}+\mathbb{D}^{e}\left(\frac{\mathrm{d} \mathbb{X}^{e}}{\mathrm{~d} t}\right)-\mathbb{F}^{e}
$$


Here $\mathbb{K}^{e}$ is the element stiffness matrix and $\mathbb{D}^{e}$ the element diffusion matrix,

$$
\mathbb{K}^{e}=\left[\begin{array}{cccc}
\mathbf{K}_{\mathbf{u} \mathbf{u}}^{e} & \mathbf{K}_{\mathbf{u} \mathbf{p}_{\mathbf{p}}}^{e} & \mathbf{K}_{\mathbf{u} \mathbf{p}_{\mathbf{f}}}^{e} & \mathbf{K}_{\mathbf{u} \mathbf{T}}^{e} \\
\mathbf{0} & \mathbf{K}_{\mathbf{p}_{\mathbf{p}} \mathbf{p}_{\mathbf{p}}}^{e} & \mathbf{K}_{\mathbf{p}_{\mathbf{p}} \mathbf{p}_{\mathbf{f}}}^{e} & \mathbf{0} \\
\mathbf{0} & \mathbf{K}_{\mathbf{p}_{\mathbf{f}} \mathbf{p}_{\mathbf{p}}}^{e} & \mathbf{K}_{\mathbf{p}_{\mathbf{f}} \mathbf{p}_{\mathbf{f}}}^{e} & \mathbf{0} \\
\mathbf{0} & \mathbf{0} & \mathbf{0} & \mathbf{K}_{\mathbf{T} \mathbf{T}}^{e}
\end{array}\right], \mathbb{D}^{e}=\left[\begin{array}{cccc}
\mathbf{0} & \mathbf{0} & \mathbf{0} & \mathbf{0} \\
\mathbf{D}_{\mathbf{p}_{\mathbf{p}} \mathbf{u}}^{e} & \mathbf{D}_{\mathbf{p}_{\mathbf{p}} \mathbf{p}_{\mathbf{p}}}^{e} & \mathbf{D}_{\mathbf{p}_{\mathbf{p}} \mathbf{p}_{\mathbf{f}}}^{e} & \mathbf{D}_{\mathbf{p}_{\mathbf{p}} \mathbf{T}}^{e} \\
\mathbf{D}_{\mathbf{p}_{\mathbf{f}} \mathbf{u}}^{e} & \mathbf{D}_{\mathbf{p}_{\mathbf{f}} \mathbf{p}_{\mathbf{p}}}^{e} & \mathbf{D}_{\mathbf{p}_{\mathbf{f}} \mathbf{p}_{\mathbf{f}}}^{e} & \mathbf{D}_{\mathbf{p}_{\mathbf{f}} \mathbf{T}}^{e} \\
\mathbf{D}_{\mathbf{T} \mathbf{u}}^{e} & \mathbf{D}_{\mathbf{T} \mathbf{p}_{\mathbf{p}}}^{e} & \mathbf{D}_{\mathbf{T} \mathbf{p}_{\mathbf{f}}}^{e} & \mathbf{D}_{\mathbf{T} \mathbf{T}}^{e}
\end{array}\right] ;
$$

while $\mathbb{F}^{e}$ is the element load vector, and $\mathbb{X}^{e}$ the element unknown vector,

$$
\mathbb{F}^{e}=\left[\begin{array}{llll}
\mathbf{R}_{\mathbf{u}}^{e} & \mathbf{R}_{\mathbf{p}_{\mathbf{p}}}^{e} & \mathbf{R}_{\mathbf{p}_{\mathbf{f}}}^{e} & \mathbf{R}_{\mathbf{T}}^{e}
\end{array}\right]^{\mathrm{T}}, \quad \mathbb{X}^{e}=\left[\begin{array}{llll}
\mathbf{u}^{e} & \mathbf{p}_{\mathbf{p}}^{e} & \mathbf{p}_{\mathbf{f}}^{e} & \mathbf{T}^{e}
\end{array}\right]^{\mathrm{T}} .
$$

All sub-matrices of $\mathbb{K}^{e}$ and $\mathbb{D}^{e}$ are listed in Appendix B. The resulting global non-linear semi-discrete equations (18) for the unknown vector $\mathbb{X}$ imply the residual $\mathbb{R}$ to vanish,

$$
\mathbb{R}=\mathbb{F}^{\text {surf }}(\mathbb{S}, \mathbb{X})-\mathbb{F}^{\text {int }}\left(\mathbb{X}, \frac{\mathrm{d} \mathbb{X}}{\mathrm{d} t}\right)=0,
$$

in which $\mathbb{F}^{\text {int }}$ is the vector of internal forces and $\mathbb{F}^{\text {surf }}$ is the vector of surface loadings denoted collectively by $\mathbb{S}$.

\subsection{Time integration}

The semi-discrete equations are integrated through a generalized trapezoidal rule defined by a scalar $\alpha \in] 0,1]$. At step $n+1$, the equations are enforced at time $t_{n+\alpha}=t_{n}+\alpha \Delta t$, with $\Delta t=t_{n+1}-t_{n}$, namely

$$
\mathbb{R}_{n+\alpha}=\mathbb{F}^{\text {surf }}\left(\mathbb{S}_{n+\alpha}, \mathbb{X}_{n+\alpha}\right)-\mathbb{F}^{\text {int }}\left(\mathbb{X}_{n+\alpha}, \mathbb{V}_{n+\alpha}\right)=0
$$

In the above relation, we define $\mathbb{Z}=\mathbb{S}, \mathbb{X}, \mathbb{V}$ as,

$$
\mathbb{Z}_{n+\alpha}=(1-\alpha) \mathbb{Z}_{n}+\alpha \mathbb{Z}_{n+1},
$$

and $\mathbb{X}_{n+1}$ and $\mathbb{V}_{n+1}$ are approximations of $\mathbb{X}\left(t_{n+1}\right)$ and $(\mathrm{d} \mathbb{X} / \mathrm{d} t)\left(t_{n+1}\right)$ respectively. The system (27) is solved iteratively by an explicit/implicit operator splitting, namely at iteration $i+1$,

$$
\mathbb{R}_{n+\alpha}^{i+1}=\mathbb{F}_{\mathrm{E}}^{\text {surf }}\left(\mathbb{S}_{n+\alpha}, \mathbb{X}_{n+\alpha}^{i}\right)-\mathbb{F}_{\mathrm{I}}^{\mathrm{int}}\left(\mathbb{X}_{n+\alpha}^{i+1}, \mathbb{V}_{n+\alpha}^{i+1}\right)=0
$$

in which,

$$
\begin{aligned}
& i=0:\left\{\begin{array}{l}
\mathbb{X}_{n+1}^{0}=\mathbb{X}_{n}+(1-\alpha) \Delta t \mathbb{V}_{n} \\
\mathbb{V}_{n+1}^{0}=\mathbb{V}_{n}
\end{array}\right. \\
& \text { for } i \geq 0:\left\{\begin{array}{l}
\mathbb{X}_{n+1}^{i+1}=\mathbb{X}_{n}+\Delta t V_{n+\alpha}^{i+1}=\mathbb{X}_{n+1}^{0}+\alpha \Delta t V_{n+1}^{i+1}=\widetilde{\mathbb{X}}_{n+1}^{i}+\alpha \Delta t \Delta \mathbb{V} \\
\mathbb{V}_{n+1}^{i+1}=\mathbb{V}_{n+1}^{i}+\Delta \mathbb{V}
\end{array}\right.
\end{aligned}
$$

where $\widetilde{\mathbb{X}}_{n+1}^{i}$ is defined as the predictor value by,

$$
i \geq 0 \quad: \quad \widetilde{\mathbb{X}}_{n+1}^{i}=\mathbb{X}_{n+1}^{0}+\alpha \Delta t \mathbb{V}_{n+1}^{i} \quad\left(=\mathbb{X}_{n+1}^{i} \quad \text { for } \quad i>0\right)
$$

and,

$$
\mathbb{X}_{n+\alpha}^{i(+1)}=(1-\alpha) \mathbb{X}_{n}+\alpha \mathbb{X}_{n+1}^{i(+1)}, \quad \mathbb{V}_{n+\alpha}^{i+1}=(1-\alpha) \mathbb{V}_{n}+\alpha \mathbb{V}_{n+1}^{i+1}
$$


The partitioning shown in the above equation (29) is motivated by two observations: 1 . the dependence of the vector of external forces on the solution is weak; 2 . the vector of internal forces depends linearly on the rate vector $\mathbb{V}$ and non-linearly on the solution $\mathbb{X}$.

A number of authors, including McTigue [3], Bear and Corapcioglu [27] and Nair et al. [17], have pointed out that the hydro-mechanical terms $T a_{p T}, T a_{f T}$ and $T c_{T} / c$ can be neglected in the energy equation (22). Indeed, these coefficients may often be of a smaller magnitude than $a_{T T}$, particularly in geotechnical materials. The rates of the primary variables in the boundary value problems addressed here have typical magnitudes, namely $\dot{p}_{p}=\dot{p}_{f} \approx O\left(10^{4}\right)$ $[\mathrm{Pa} / \mathrm{s}], \dot{u} \approx O\left(10^{-6}\right)[\mathrm{m} / \mathrm{s}]$ and $\dot{T} \approx O(10)[\mathrm{K} / \mathrm{s}]$. Therefore, by using Table 1 ,

$$
\left.\begin{array}{l}
T a_{p T} \dot{p}_{p} \approx O\left(10^{1}\right) \\
T a_{f T} \dot{p}_{f} \approx O\left(10^{0}\right) \\
T c_{T} / c \operatorname{tr} \dot{\boldsymbol{\epsilon}} \approx O\left(10^{1}\right)
\end{array}\right\}<<a_{T T} \dot{T} \approx O\left(10^{5}\right)\left[\mathrm{W} / \mathrm{m}^{3}\right] .
$$

Nevertheless, all terms are preserved in this formulation for the sake of generality.

The global iteration process uses the full Newton-Raphson procedure as described above. The equation system to be solved is obtained by insertion of the time-integrator (30)-(32) in the residual (29),

$$
\mathbb{R}_{n+\alpha}^{i+1}=\mathbb{R}_{n+\alpha}^{i}-\mathbb{C}^{*} \alpha \Delta \mathbb{V}=0 \quad \Leftrightarrow \quad \mathbb{C}^{*}(\alpha \Delta \mathbb{V})=\mathbb{R}_{n+\alpha}^{i},
$$

in which the Newton direction $\Delta \mathbb{V}$ is sought. The effective diffusion matrix $\mathbb{C}^{*}$ can be expressed in terms of the global diffusion matrix $\mathbb{D}$ and the global stiffness matrix $\mathbb{K}$,

$$
\mathbb{C}^{*}=\underbrace{\left(\frac{\partial \mathbb{F}_{I}^{\mathrm{int}}}{\partial \mathbb{V}}\right)\left(\widetilde{\mathbb{X}}_{n+\alpha}^{i}\right)}_{\mathbb{D}}+\underbrace{\left(\frac{\partial \mathbb{F}_{I}^{\mathrm{int}}}{\partial \mathbb{X}}\right)\left(\widetilde{\mathbb{X}}_{n+\alpha}^{i}\right)}_{\mathbb{K}} \alpha \Delta t
$$

where it has been recognized that the derivatives of $\mathbb{F}_{I}^{\text {int }}$ depend linearly on $\mathbb{V}$ so that the stiffness matrix $\mathbb{K}$, and the diffusion matrix $\mathbb{D}$ do not depend on $\mathbb{V}$ but only on $\mathbb{X}$.

The time step $\Delta t$ is increased from 1 to $1000 \mathrm{~s}$ in order to keep the number of computer time to a reasonable value. The time integration parameter $\alpha$ is taken equal to $2 / 3$ corresponding to the Galerkin method that provides unconditional stability and first order of accuracy, for linear problems and a single pass. Experience has shown that $\alpha$ equal to $2 / 3$ provides better stability than the Crank-Nicolson method, $\alpha$ equal to $1 / 2$, in spite of the lower accuracy. With $\alpha=1 / 2$, the profile of the solution displays some oscillations, particularly in the vicinity of the perturbation [28], p. 467. The four-node element (QUAD4) is used to interpolate all unknowns. The number of integration points is equal to two (in each spatial dimension), for all stiffness matrices and all residuals. The finite element code has been developed as part of this research.

\section{Non-isothermal borehole stability analysis}

The thermo-hydro-mechanical constitutive equations are now used to assess the stability of a borehole in a heavy-oil recovery context, i.e. a thermally stimulated reservoir.

To this end, a vertical wellbore drilled in a thermo-poro-elastic fractured medium is considered, see Figure 1. A unit thickness of the formation $(h=1 \mathrm{~m})$ is analyzed in which, the inner radius $r_{1}$ is set to $0.1 \mathrm{~m}$ and the far-field radius $r_{2}$ is set to $800 \mathrm{~m}$ to represent a boundary at a large distance. 
We denote the initial (prior to drilling) pore and fissure pressures as $p_{p}^{0}$ and $p_{f}^{0}$; and the initial temperature as $T^{0}$. The fully saturated formation is located at a depth of $1000 \mathrm{~m}$ and is subjected to the following vertical gradients of in situ stresses, fluid pressures and temperature,

$$
\begin{aligned}
& \partial \sigma_{z}^{0} / \partial z=-29.0 \mathrm{kPa} / \mathrm{m} \\
& \partial \sigma_{x}^{0} / \partial z=\partial \sigma_{y}^{0} / \partial z=-23.5 \mathrm{kPa} / \mathrm{m} \\
& \partial p_{p}^{0} / \partial z=\partial p_{f}^{0} / \partial z=9.8 \mathrm{kPa} / \mathrm{m} \\
& \partial T^{0} / \partial z=0.05^{\circ} \mathrm{C} / \mathrm{m} .
\end{aligned}
$$

The fractured reservoir is idealized as a dual porosity medium with the isotropic material properties presented in Table 1. Since both the loading conditions display symmetry about the vertical axis of the wellbore an axi-symmetric formulation is employed. To reduce computational time without loss of accuracy, a graded mesh is used and the size of the finite elements is increased along the radial direction (towards $r_{2}$ in Figure 1) according to a logarithmic rule.

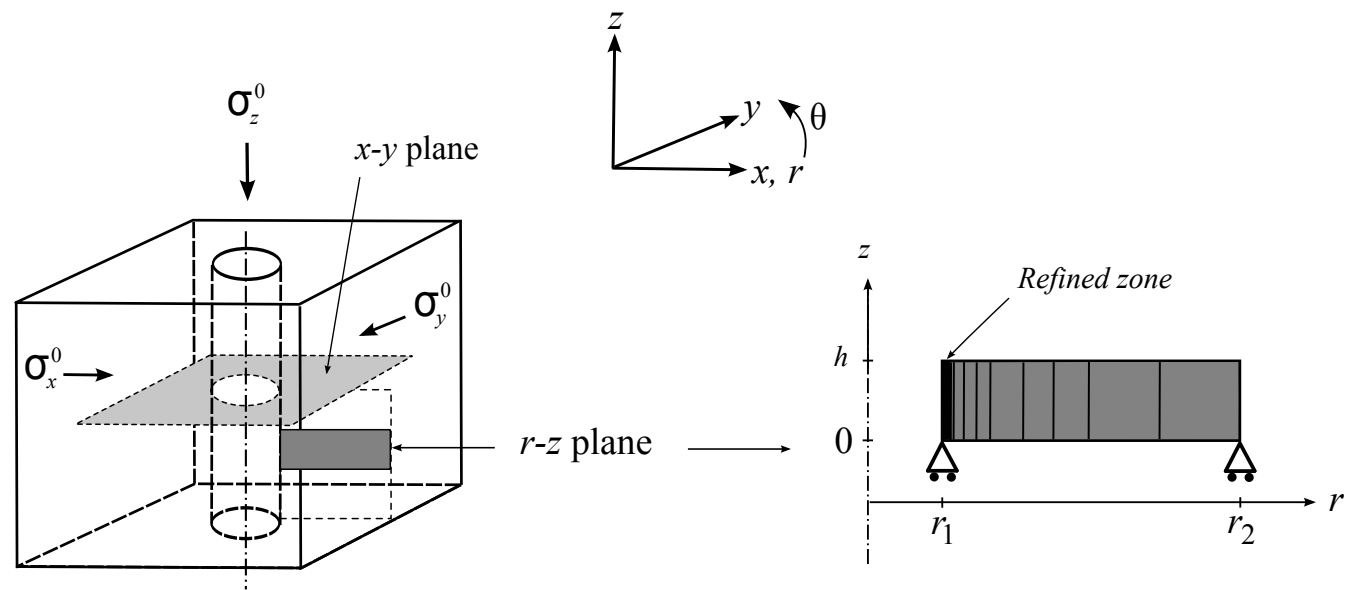

Figure 1: Schematic diagram of a vertical borehole subjected to in situ stresses (left). 2D representation of the problem with an axi-symmetric mesh in the $r-z$ plane (right).

The cap rock on the top of the reservoir is considered to be rigid, constraining the vertical deformation of the reservoir. The initial vertical strain, which represents the deformation experienced by the reservoir prior to drilling,

$$
\epsilon_{z}^{0}=-\frac{\nu}{E}\left(\sigma_{x}^{0}+\sigma_{y}^{0}\right)+\frac{1}{E} \sigma_{z}^{0}+\frac{c_{p}-c_{s}}{3} p_{p}^{0}+\frac{c-c_{p}}{3} p_{f}^{0}+\underbrace{\frac{c_{T}}{3}\left(T^{0}-T^{0}\right)}_{=0},
$$

is obtained from equation (7).

\subsection{Boundary conditions}

The boundary conditions at the far-field radius $r=r_{2}$, at the top $z=h$ and at the bottom $z=0$ of the dual porous medium are presented in Table 2 . To test the stability of the borehole, a constant mud pressure $p_{w}=12.0 \mathrm{MPa}$ is applied. The boundary conditions on the wellbore radius $r=r_{1}$ are handled separately according to two distinct configurations.

Indeed, two types of conditions are envisaged at the dual-porosity wellbore wall $r=r_{1}$ : a fully permeable boundary condition on both cavities and a semi-permeable boundary condition in which the porous blocks are sealed by the drilling mud cake and have zero outward flux. 


\begin{tabular}{lccc}
\hline \hline Material parameter & Value & Unit & Ref. \\
\hline Elastic modulus $E$ & 9.5 & $\mathrm{GPa}$ & 1 \\
Poisson's ratio $\nu$ & 0.25 & - & 1 \\
Bulk modulus of grains $K_{s}=1 / c_{s}$ & 10 & $\mathrm{GPa}$ & 1 \\
Bulk moduli of the fluids $K_{k}=1 / c_{H, k}$ & 4.3 & $\mathrm{GPa}$ & 1 \\
Viscosities of the fluids $\mu_{p}=\mu_{f}$ & $10^{-3}$ & $\mathrm{~Pa} . \mathrm{s}$ & 1,2 \\
Porous blocks porosity $n_{p}$ & 0.15 & - & 1,2 \\
Porous blocks permeability $k_{p}$ & $5 \times 10^{-20}$ & $\mathrm{~m}^{2}$ & 1,2 \\
Compressibility ratio $c_{p} / c$ & 0.9 & - & 3 \\
Fissure network porosity $n_{f}$ & $0.1 \times n_{p}$ & - & 3 \\
Fissure network permeability $k_{f}$ & $10 \times k_{p}$ & $\mathrm{~m}{ }^{2}$ & 3 \\
Thermal conductivity of the porous medium $\lambda$ & 2.65 & $\mathrm{~W} / \mathrm{m} . \mathrm{K}$ & 1 \\
Volumetric thermal expansion of the porous medium $c_{T}$ & $1.8 \times 10^{-5}$ & $1 / \mathrm{K}$ & 1,2 \\
Volumetric thermal expansion of the fluids $c_{T, p}=c_{T, f}$ & $4.5 \times 10^{-4}$ & $1 / \mathrm{K}$ & 4 \\
Heat capacity of the porous medium $C_{v}$ & 837 & $\mathrm{~J} / \mathrm{kg} . \mathrm{K}$ & 1,2 \\
Density of the porous medium $\rho$ & 1980 & $\mathrm{~kg} / \mathrm{m}^{3}$ & 1,2 \\
\hline \hline
\end{tabular}

Table 1: Input parameters representative of a homogeneous isotropic dual porous medium. 1: Nair et al. [18], 2: Nair et al. [17] Gulf of Mexico Shale, 3: Wilson and Aifantis [29] and 4: Kestin [30] p. 541.

\begin{tabular}{l|l}
\hline \hline$r=r_{2}$ & $r \in] r_{1}, r_{2}[$ \\
$z \in[0, h]$ & $z=h$ and $z=0$ \\
\hline$\sigma_{r}\left(r_{2}, z\right)=\sigma_{r}^{0}$ & $\epsilon_{z}(r, z)=\epsilon_{z}^{0}$ \\
$p_{p}\left(r_{2}, z\right)=p_{p}^{0}$ & $\mathbf{J}_{p}(r, z)=\mathbf{0}$ \\
$p_{f}\left(r_{2}, z\right)=p_{f}^{0}$ & $\mathbf{J}_{f}(r, z)=\mathbf{0}$ \\
$T\left(r_{2}, z\right)=T^{0}$ & $\mathbf{q}(r, z)=\mathbf{0}$ \\
\hline \hline
\end{tabular}

Table 2: Boundary conditions for a thermal recovery test with an axi-symmetric mesh in the $r-z$ plane.

a) Permeable boundary. The radial stress, the pressures of the fluid in the two cavities and the temperature are considered to be totally controlled at the wellbore, for any $z \in[0, h]$,

$$
\begin{aligned}
\sigma_{r}\left(r_{1}, z\right) & =\sigma_{r}^{0} H(-t)-p_{w} H(t), \\
p_{p}\left(r_{1}, z\right) & =p_{p}^{0} H(-t)+p_{w} H(t), \\
p_{f}\left(r_{1}, z\right) & =p_{f}^{0} H(-t)+p_{w} H(t), \\
T\left(r_{1}, z\right) & =T^{0} H(-t)+T_{w} H(t),
\end{aligned}
$$

where $H(t)$ is the Heaviside step function (Figure 2).

b) Semi-permeable boundary. In this case a mud cake is formed on the borehole wall as the drilling mud infiltrates into the permeable medium. Therefore, a pore matrix endowed with low permeability can be sealed at the inlet by the mud cake. We term this boundary condition a semi-permeable boundary, since the sealing process is applied only to the porous blocks (Figure 2). The second relationship in (38) is therefore changed to,

$$
\mathbf{J}_{p}\left(r_{1}, z\right)=\mathbf{0} .
$$



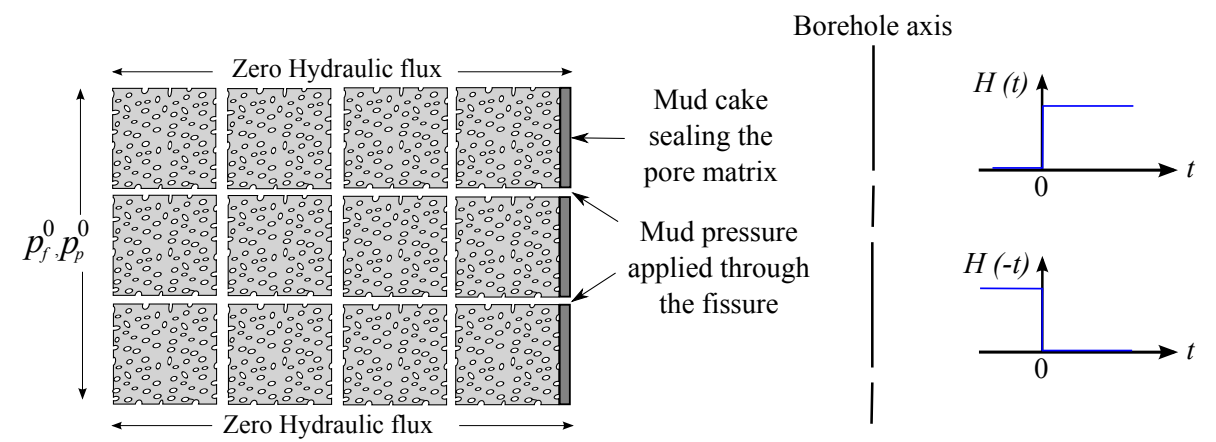

Figure 2: Sketch of a semi-permeable hydraulic boundary condition with zero flux at the porous blocks boundary.

The overall compressibility of the mixture $c$ and the Lamé constants of the drained solid $\lambda$ and $\mu$, are deduced from the Young's modulus $E$ and Poisson's ratio $\nu$ through the elastic relationships,

$$
c=3 \frac{1-2 \nu}{E}, \quad \lambda=\frac{E \nu}{(1+\nu)(1-2 \nu)}, \quad \mu=\frac{E}{2(1+\nu)} .
$$

\subsection{Characteristic parameters}

In a porous medium with a single porosity, the pressure response to a heating/cooling test is controlled by the diffusivity ratio $R=\sqrt{\alpha_{H} / \alpha_{T}}$ of the hydraulic diffusivity $\alpha_{H}$ over the thermal diffusivity $\alpha_{T}$ defined by McTigue [3],

$$
\alpha_{T}=\frac{\Lambda}{\rho C_{v}}, \quad \alpha_{H}=\frac{k_{p}}{\mu_{p}} \frac{2 \mu(1-\nu)}{1-2 \nu} \frac{B^{2}\left(1+\nu_{u}\right)^{2}(1-2 \nu)}{9\left(1-\nu_{u}\right)\left(\nu_{u}-\nu\right)}
$$

in which $B$ is the Skempton coefficient and $\nu_{u}$ is the undrained Poisson's ratio,

$$
\frac{1}{B}=1+n_{p} \frac{c_{H, p}-c_{s}}{c-c_{s}}, \quad \nu_{u}=\frac{3 \nu+B(1-2 \nu)\left(1-c_{s} / c\right)}{3-B(1-2 \nu)\left(1-c_{s} / c\right)}
$$

In a dual porosity medium (2P), hydraulic diffusion takes place in two spatially separated cavities, and as such two distinct diffusivity ratios can be defined. For the porous blocks, $R$ is typically smaller than one, $R<1$ with the thermal diffusion being faster than the hydraulic diffusion. Conversely, for the fissure network $R$ is greater than one, $R>1$ in which the hydraulic flow is faster than the heat flow. Crucial to the understanding of the behavior is the fact that the porous blocks and the fissure network are endowed with opposite characteristics of diffusion dominance.

For the sake of comparison, the diffusivity ratio of the associated single porous medium (A1P) is also analyzed (Table 3 ). For this case, the overall porosity is defined as $n_{\mathrm{A} 1 \mathrm{P}}=n_{p}+n_{f}$ and the overall permeability as $k_{\mathrm{A} 1 \mathrm{P}}=k_{p}+k_{f}$. This single porous medium is endowed with a single unknown pressure For this single porous medium (A1P), the thermal diffusivity front is behind the hydraulic diffusion front with $R_{\mathrm{A} 1 \mathrm{P}}=2.16$, analogous to the fissure network characteristic.

In addition to the diffusivity ratios, the responses of the dual porosity mixture are scrutinized with the help of two other characteristic parameters, namely, a characteristic time $t^{*}$ and a characteristic leakage parameter $\eta^{*}$. Both parameters are defined with respect to a point of interest $\left.r_{c} \in\right] r_{1}, r_{2}$ [ in the vicinity of the borehole. This point, which defines the boundary of the failure zone, is chosen as equal to ten times the radius of the borehole, $r_{c}=1 \mathrm{~m}$. 


\begin{tabular}{lccc}
\hline \hline Parameter & Pore matrix (2P) & (A1P) & Fissure network (2P) \\
\hline Porosity, $n_{k}(-)$ & 0.14 & 0.154 & 0.014 \\
Permeability, $k_{k}\left(\mathrm{~m}^{2}\right)$ & $5 \times 10^{-20}$ & $5.5 \times 10^{-19}$ & $5 \times 10^{-19}$ \\
Hydraulic diffusivity, $\alpha_{H}\left(\mathrm{~m}^{2} / \mathrm{s}\right)$ & $6.97 \times 10^{-7}$ & $7.48 \times 10^{-6}$ & $9.06 \times 10^{-6}$ \\
Thermal diffusivity, $\alpha_{T}\left(\mathrm{~m}^{2} / \mathrm{s}\right)$ & $1.6 \times 10^{-6}$ & $1.6 \times 10^{-6}$ & $1.6 \times 10^{-6}$ \\
Diffusivity ratio, $R(-)$ & $0.66<$ & $1<2.16$ & $<2.38$ \\
\hline \hline
\end{tabular}

Table 3: Comparison between the diffusivity ratios $R=\sqrt{\alpha_{H} / \alpha_{T}}$ of the porous blocks, of the fissure network as parts of dual porosity medium, and of the associated single porosity medium. Note that thermal diffusion is faster than hydraulic diffusion for the pore matrix $(2 \mathrm{P})$ while the opposite holds for the fissure network $(2 \mathrm{P})$ and foe the associated single porosity analysis (A1P).

A characteristic time defined as $t^{*}=\left(r_{c}-r_{1}\right)^{2} / \alpha$ represents the time that is required for a diffusion front to reach the point of interest $r_{c}$ in which $\alpha$ is a diffusion parameter. For a region of length $r_{c}-r_{1}$, the diffusion time scale distinguishes the early time from the late time responses of the system. The analysis of thermo-hydro-mechanical loading in dual porous media highlights three characteristic times, namely, the end of hydraulic diffusion through the fissures at time $8.17 \times 10^{4} \mathrm{~s}$, second the end of thermal diffusion in the mixture at time $5.07 \times 10^{5} \mathrm{~s}$, and last the end of hydraulic diffusion through the pores at time $1.4 \times 10^{6} \mathrm{~s}$. We chose the characteristic time of the system to be the largest of these three characteristic times; therefore, at $t=t^{*}$, all the diffusion fronts should have reached $r_{c}$.

The dimensionless characteristic leakage parameter $\eta^{*}$ is expressed as in [26],

$$
\eta^{*}=\eta \quad \frac{\mu_{p} r_{c}^{2}}{k_{p}+k_{f}}
$$

in which $\mu_{p}=\mu_{f}$ due to thermal equilibrium. For an average fracture spacing $l$ equal to $0.03 \mathrm{~m}$, one set of fractures $n=1$ and the material parameters presented in Table 1 , the average characteristic leakage parameter is, according to eqn (43), equal to $\eta_{a v}^{*}=1.1 \times 10^{3}$.

\section{Thermal effects on dual porous media}

Numerical results of thermal effects in the vicinity of the borehole in a fractured reservoir are now presented (Table 1). Two sensitivity analyses are carried out: the influences of the temperature loading $\Delta T=T_{w}-T^{0}$ and of the leakage parameter $\eta$ on the pressure and stress responses of the system are examined. The variation with time of the effective stresses around the borehole are scrutinized and a stability analysis is conducted at the most unfavorable time.

Results are presented from Figures 3 to 10. A continuum mechanics convention is used with compressive stresses taken as negative.

\subsection{Influence of temperature}

The effect of thermal loading on a single porosity model has been analyzed by McTigue [3] who provides analytical responses to a half-plane subjected either to a jump of heat flux or to a jump of temperature. These analytical responses have been used to examine the response of the finite element code in the particular case of a single porosity model.

In what follows, the effect of temperature on a fractured medium is presented and the features of the dual porosity approach are compared with a standard single porosity model. 
Both heating and cooling tests are performed. The difference between the temperature of the fluid at the wellbore $T_{w}$ and the temperature of the in situ fluid $T^{0}$ is successively set to $+50{ }^{\circ} \mathrm{C}$, $-50^{\circ} \mathrm{C}$ and $0^{\circ} \mathrm{C}$. The leakage parameter is set equal to the average leakage parameter $\eta^{*}=\eta_{a v}^{*}$. The results are presented at time $t=80 \mathrm{~s}$, which corresponds to an early time response of the system and where the difference between the three loadings is the largest.
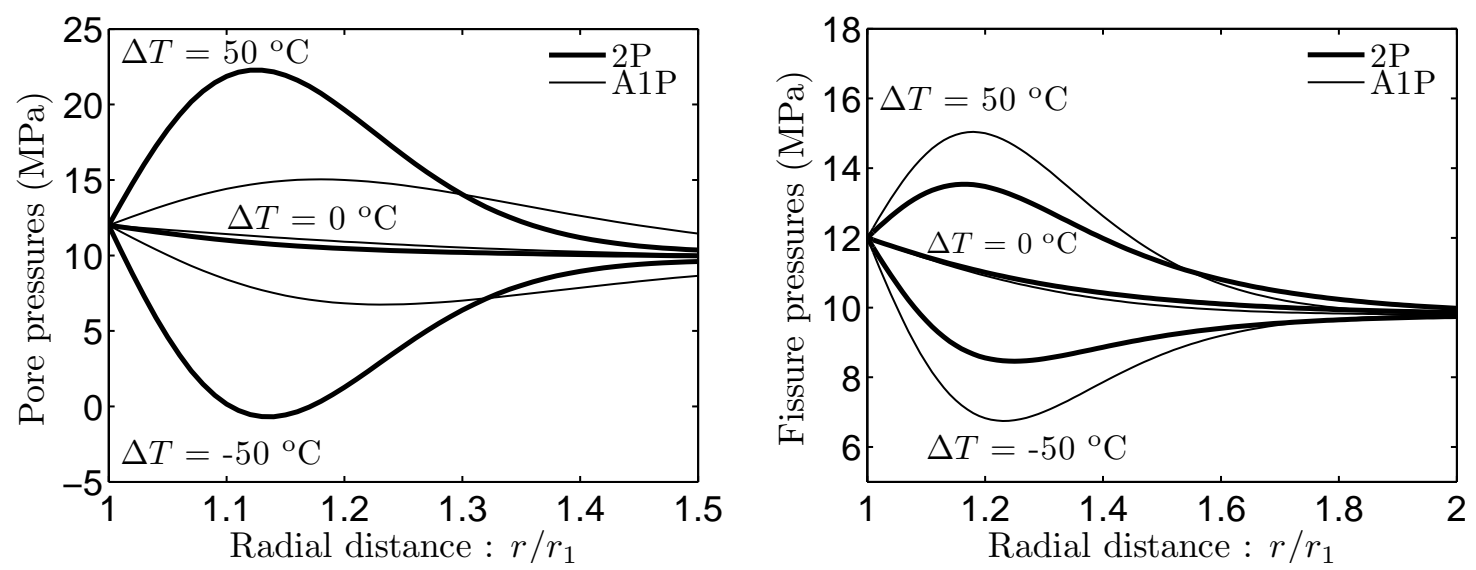

Figure 3: Profiles of pore pressure and fissure pressure at time $80 \mathrm{~s}$ and for three imposed temperature changes $\Delta T$ at the borehole. The leakage parameter is set equal to the average leakage parameter $\eta^{*}=\eta_{a v}^{*}$.

The pore pressure induced by thermal stimulation is due to distinct thermal expansion coefficients of the solid skeleton and that of the fluid (Table 1). The general trend is that heating, $T_{w}-T^{0}=50^{\circ} \mathrm{C}$, induces a higher pore pressure and therefore a lower radial effective stress than $T_{w}-T^{0}=0{ }^{\circ} \mathrm{C}$. Cooling, $T_{w}-T^{0}=-50^{\circ} \mathrm{C}$, induces the opposite response (Figure 3-4). A hot thermal stimulation weakens the wellbore, whereas cooling shields against failure.

When considering the two cavities as part of a dual porous medium, the pressure responses (2P) are significantly different from the associated single porosity model (A1P). As expected, such behavior is due to the disparity between the diffusivity ratio of the porous blocks $R_{p}=0.66$ and the diffusivity ratio of the fissure network $R_{f}=2.38$. In the porous blocks, thermal diffusion is faster than hydraulic diffusion and therefore the pore pressure is unable to dissipate, causing changes of fluid pressure. On the other hand, in the fissure network, the thermally induced pressure dissipates as soon as it is generated.

The effective stresses displayed in Figure 4 represent the stresses undergone by the solid skeleton. They are defined by Biot's relationship extended to dual porosity [19],

$$
\boldsymbol{\sigma}^{\prime}=\boldsymbol{\sigma}+\xi_{p} p_{p} \mathbf{I}+\xi_{f} p_{f} \mathbf{I} .
$$

With the material parameters presented in Table 1, the effective stress coefficients $\xi_{p}$ and $\xi_{f}$ are equal to 0.27 and 0.1 respectively. Again, compared with the associated single porosity model, the dual porosity approach displays a wider range of effective stress variations related to the pore pressure response (eqn 44). The associated single porosity model significantly underestimates the changes of fluid pressures and effective stresses.

\subsection{Influence of mass transfer}

In a dual porosity medium, the mass transfer coefficient influences significantly the profiles of the fluid pressures and of the effective stresses. A parameter analysis is now conducted to examine the influence of mass transfer and to identify when the dual porosity approach becomes essentially identical to a classical single porosity model. 

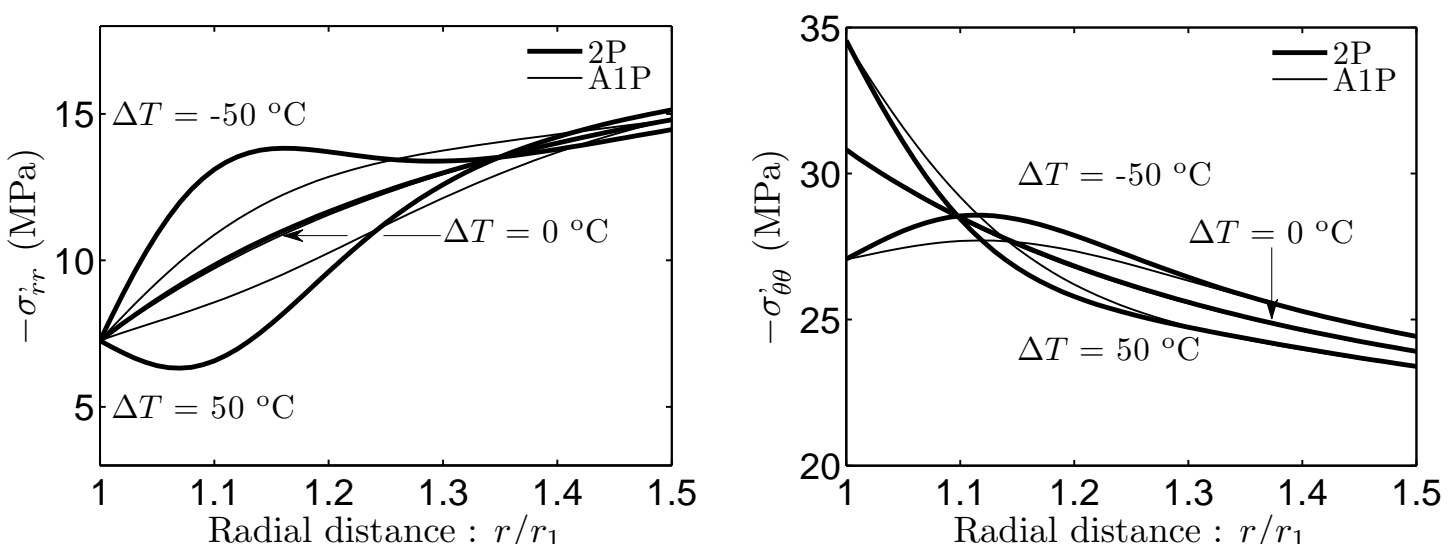

Figure 4: Same as Figure 3. Profiles of radial effective stress and tangential effective stress, at time $80 \mathrm{~s}$ and for three imposed temperature changes $\Delta T$ at the borehole.

The characteristic leakage parameter $\eta_{e q}^{*}$ corresponds to the minimum dimensionless mass transfer coefficient that delivers instantaneous hydraulic equilibrium at a time of interest. For $t=80 \mathrm{~s}, \eta_{e q}^{*}$ is equal to $1.1 \times 10^{6}$. This value was found by trial and error and is specific to the dual porous medium characterized in Table 1 . Note that $\eta_{e q}^{*}$ is a time-dependent parameter.

The influence of mass transfer is illustrated by selecting three representative values of the leakage parameter: $\eta^{*} / \eta_{e q}^{*}=0$ which represents no mass transfer, $\eta^{*} / \eta_{e q}^{*}=0.001$ which represents a low mass transfer, and $\eta^{*} / \eta_{e q}^{*} \geq 1$ which corresponds to instantaneous hydraulic equilibrium. The leakage parameter is related to the average fissure spacing and to the porous blocks permeability, and hence represents the ability of the dual porous medium to transfer fluid mass from one cavity to the other. In this section and hereafter, only heating tests $\Delta T=+50^{\circ} \mathrm{C}$ will be considered.

The sensitivity of the fluid pressures to the leakage parameter is presented in Figure 5 for two distinct types of boundary condition: (left) permeable boundary and (right) a semi-permeable boundary. A comparison with an associated single porous medium is also presented.

In general, the pore pressure increases due to a combination of low permeability and higher thermal expansion for the fluid than for the solid, whereas the fissure pressure dissipates rapidly due to a higher permeability. For $\eta^{*} / \eta_{e q}^{*}=0$, the two fluid fields are de-coupled and no mass transfer occurs. For $\eta^{*} / \eta_{e q}^{*}=0.001$, moderate mass transfer takes place as the pore pressure dissipates into the fissures. Consequently, the pore pressure peak in the porous blocks is lower than for $\eta^{*} / \eta_{e q}^{*}=0$, and the pressure in the fissure network is higher than the associated single porosity response. Finally, for $\eta^{*} / \eta_{e q}^{*} \geq 1$, the mass transfer between the pores and the fissures is instantaneous and the two cavities are in hydraulic equilibrium. Note that this equilibrium pressure is not equal to the fissure pressure when $\eta^{*} / \eta_{e q}^{*}=0$, but is equal to the pressure response of the associated single porous medium. For $\eta^{*} / \eta_{e q}^{*} \geq 1$, the pore pressure, the fissure pressure and the pore pressure of the associated single porosity model superpose.

For a permeable boundary, the pore pressure maximum is located a short distance from the wellbore wall due to a flux inward to the formation $\mathbf{J}_{p} \geq 0$ for radii larger than $r / r_{1} \geq 1.2$ and a flux outward to the wellbore $\mathbf{J}_{p} \leq 0$ for radii smaller than $r / r_{1} \leq 1.1$. For a semi-permeable boundary, the maximum is located at the borehole itself due to the flux of the porous blocks $\mathbf{J}_{p}=0$, which induces a zero pore pressure gradient $\nabla p_{p}=0$ at $r=r_{1}$. In addition, the magnitude of the pore pressure maximum is higher for the semi-permeable boundary than for the permeable boundary since the pore fluid can only dissipate internally and through mass 
transfer. The pore pressure peak is smaller for the permeable boundary due to the direct dissipation the pressure through the wellbore wall.

Permeable boundary

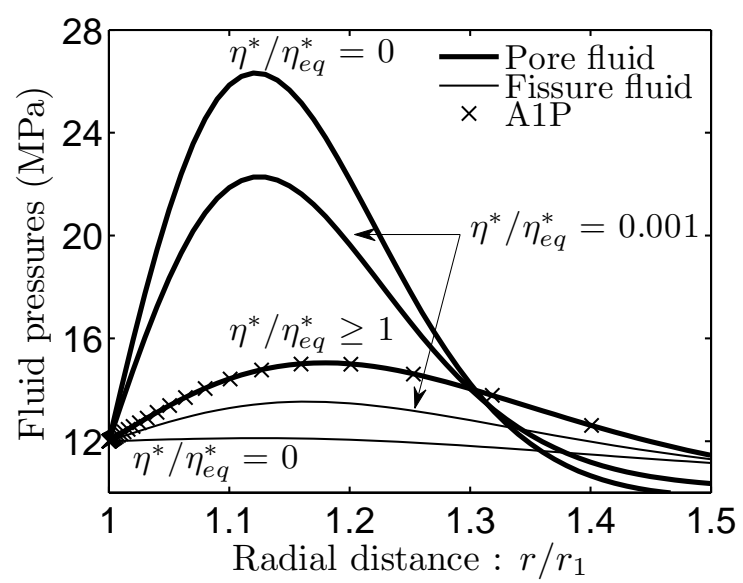

Semi-permeable boundary

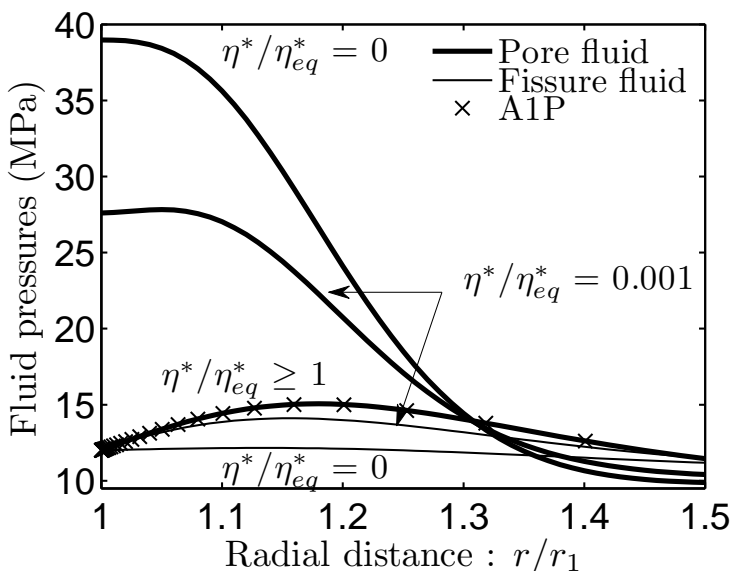

Figure 5: Profiles of pore pressure and fissure pressure, at time $80 \mathrm{~s}$, for a thermal loading equal to $T_{w}-T^{0}=50^{\circ} \mathrm{C}$.
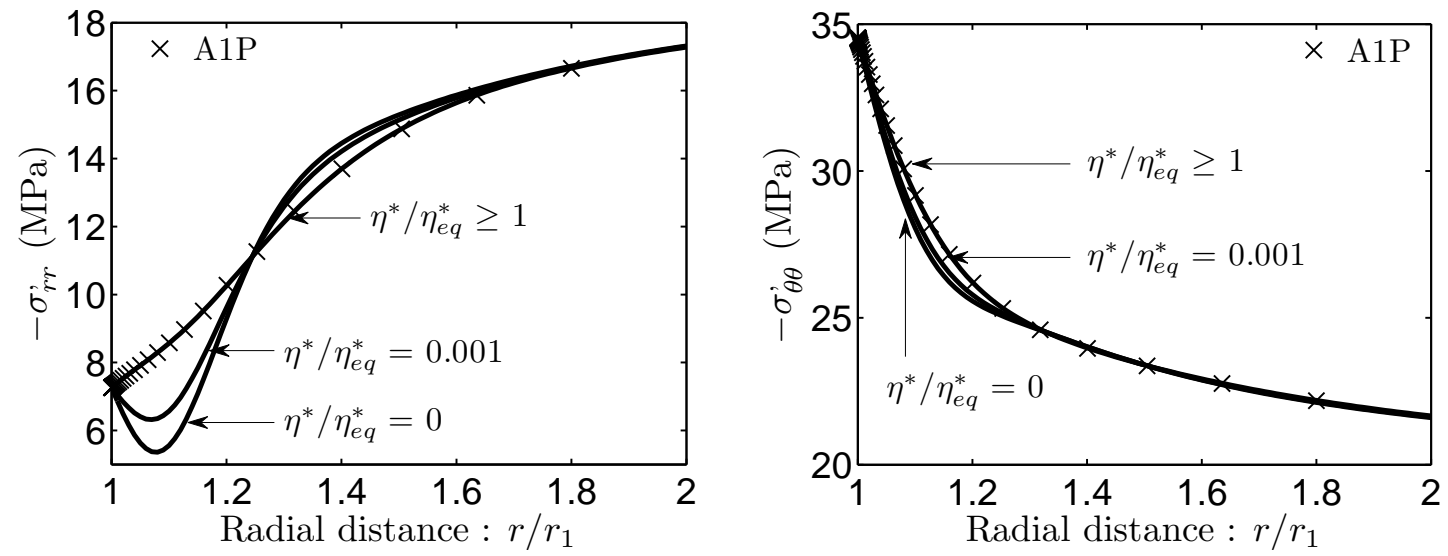

Figure 6: Profiles of effective radial stress and effective tangential stress, at time $80 \mathrm{~s}$, accounting for a permeable boundary, for a thermal loading equal to $T_{w}-T^{0}=50^{\circ} \mathrm{C}$.

The sensitivity of the effective stresses to the mass transfer coefficient is illustrated in Figures 6 and 7. The compressive effective radial stress experiences a significant drop for $\eta^{*} / \eta_{e q}^{*}=0$, which tends to diminish for high mass transfer $\eta^{*} / \eta_{e q}^{*} \geq 1$. By comparing Figures 6 and 7 , the semi-permeable boundary is seen to introduce a greater reduction in effective radial stress than the permeable boundary. For a low leakage parameter (close to zero), the effective radial stress shifts from compressive to tensile. The positive effect of the compressive mud pressure applied at the borehole is annihilated by the thermally induced increase in pore fluid pressure.

Mass transfer from the porous blocks to the fissure network increases the dissipation of the pore pressure and conversely decreases the dissipation of the fissure pressure. This in turn increases the apparent diffusivity ratio of the pore matrix and decreases the apparent diffusivity ratio of the fissure network. For the intermediate leakage parameters $\eta^{*} / \eta_{e q}^{*} \leq 0.1$, the dual porosity approach is the only one which can represent correctly the reduction in the effective radial stress induced by the pore pressure response. 

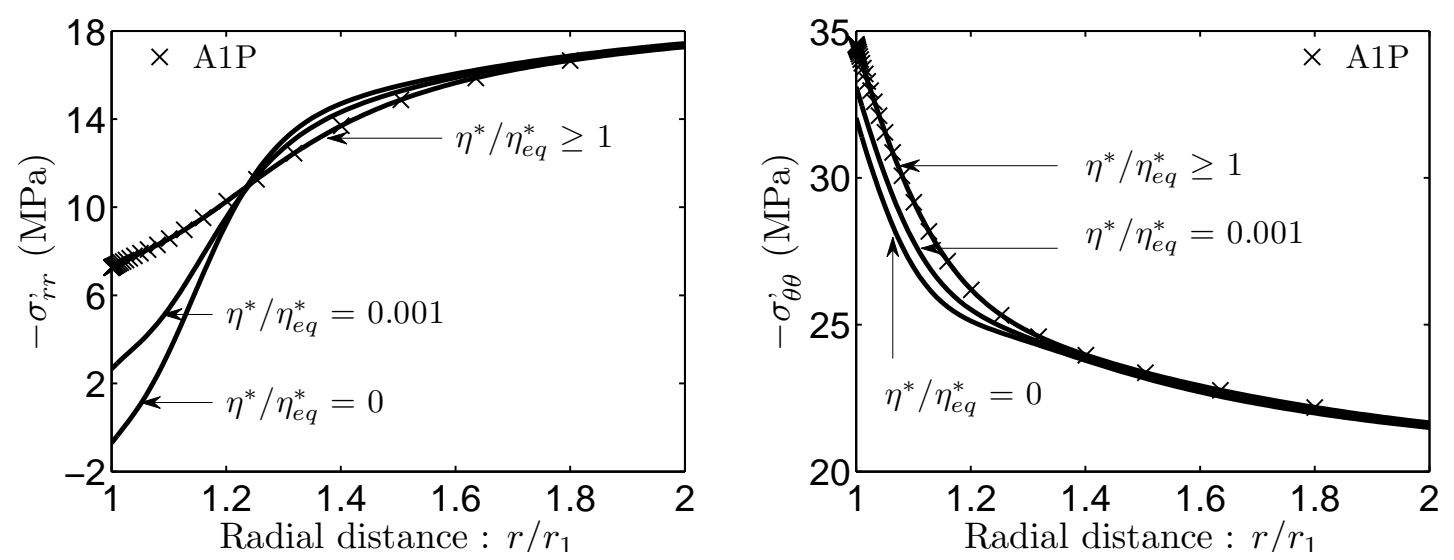

Figure 7: Same as Figure 6 for a semi-permeable boundary.

\subsection{Time profiles}

The variation with time of the fluid pressures gives an indication of the time scale at which the dual porosity model has the largest influence on the effective stress response. The pressure and stress profiles are shown for three representative times, namely at the very short time $t=6 \mathrm{~s}$ corresponding to $t / t^{*}=4.210^{-6}$, at the short time $t=80 \mathrm{~s}$ corresponding to $t / t^{*}=5.510^{-5}$ and finally at the intermediate time $t=800 \mathrm{~s}$ corresponding to $t / t^{*}=5.510^{-4}$. Because the characteristic time $t^{*}$ is related to the slowest diffusion phenomenon (and does not account for mass transfer effects), the ratio $t / t^{*}$ remains small even for the intermediate time. Focusing on a permeable boundary at the wellbore and on a heating test $T_{w}-T^{0}=50^{\circ} \mathrm{C}$, the results are presented for two values of the dimensionless leakage parameter representing an infinite fracture spacing, $\eta^{*}=0$, and an average fracture spacing equal to $0.03 \mathrm{~m}, \eta^{*}=\eta_{a v}^{*}$.

Over time, the pore pressure front propagates into the formation away from the borehole with a diminishing pressure peak (Figure 8). As expected, the larger the leakage parameter, the faster the dissipation of the pore pressure. Contrary to the pore pressure behavior, the pressure in the fissure network dissipates more slowly with an increase in the leakage parameter. Note that for the very short time response $(t=6 \mathrm{~s})$, average mass transfer does not play a significant role in the fluid pressure response of the system and pore and fissure fluid pressures, for $\eta^{*}=0$ and $\eta^{*}=\eta_{a v}^{*}$, differ little.

As time elapses, the effective radial stress becomes more compressive as a consequence of diffusion (Figure 9). At the time $t=6 \mathrm{~s}$, the effective radial stress displays the highest compressive reduction due to the slow dissipation of the pore pressure. Similarly, there is an uneven reduction in the compressive effective tangential stress at very short time. For both effective stresses at $t=6 \mathrm{~s}$ and $t=800 \mathrm{~s}$, the two curves $\eta^{*}=0$ and $\eta^{*}=\eta_{a v}^{*}$ are superposed. Over time, the effective tangential stress becomes less compressive while the effective radial stress behaves conversely. The time required for the effective stresses to attain steady state is directly related to the mass transfer coefficient.

At $t=6 \mathrm{~s}$, mass transfer and dissipation have not taken place yet. At the intermediate time $t=800 \mathrm{~s}$, hydraulic equilibrium has almost been reached for the average mass transfer value $\eta^{*}=\eta_{a v}^{*}$; however, the influence of the diffusion and the mass transfer in the form of a difference in pore and fissure pressures is still prominent at $t=80 \mathrm{~s}$. The reduction in compressive radial effective stress is maximum at $t=6 \mathrm{~s}$, and hence the borehole failure analysis is most critical at the very short time scale. 

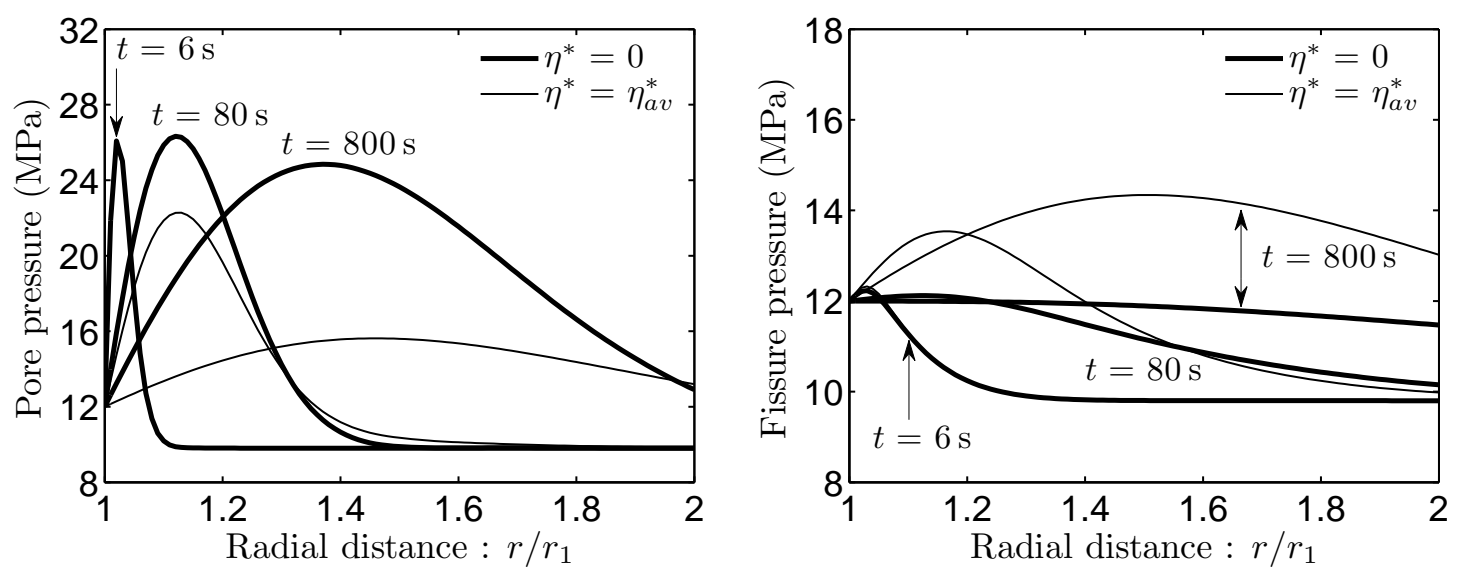

Figure 8: Profiles of pore pressure and fissure pressure, at three representative times, for two values of the leakage parameter $\eta^{*}=0$ and $\eta^{*}=\eta_{a v}^{*}$.
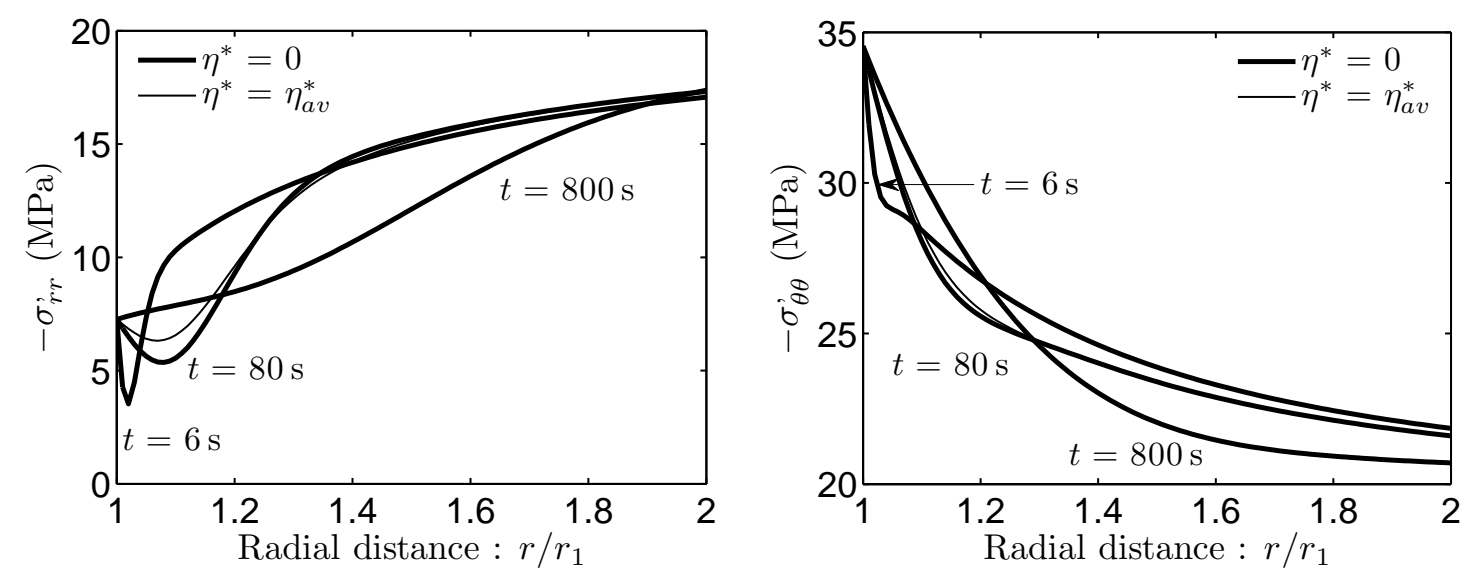

Figure 9: Same as Figure 8. Profiles of effective radial stress and effective tangential stress.

\subsection{Borehole stability analysis}

The rise in pore water pressure plays a key role in the stability analysis of boreholes. Failure typically occurs when the pore pressure reduces the effective confining pressure below a critical value [5]. Thus, for an accurate failure prediction, a general understanding of the coupled thermo-hydro-mechanical response of the effective stress, governed by Biot's poroelastic law extended to dual porous media eqn (44), is required [19].

To illustrate the borehole stability analysis, we present the results in the form of the effective stress path along a radial direction away from the wellbore. The stress path is phrased in terms of the mean effective stress $p^{\prime}$ and shear stress $q$. The mean effective stress $p^{\prime}$ and the shear stress $q$ are, respectively, equal to,

$$
p^{\prime}=-\frac{1}{3}\left(\sigma_{r r}^{\prime}+\sigma_{\theta \theta}^{\prime}+\sigma_{z z}^{\prime}\right),
$$

and,

$$
q^{2}=\frac{1}{6}\left(\left(\sigma_{r r}^{\prime}-\sigma_{\theta \theta}^{\prime}\right)^{2}+\left(\sigma_{\theta \theta}^{\prime}-\sigma_{z z}^{\prime}\right)^{2}+\left(\sigma_{z z}^{\prime}-\sigma_{r r}^{\prime}\right)^{2}\right)+\left(\sigma_{r \theta}^{\prime}\right)^{2}+\left(\sigma_{r z}^{\prime}\right)^{2}+\left(\sigma_{\theta z}^{\prime}\right)^{2} .
$$


To perform the borehole collapse analysis, the Drucker-Prager criterion [31] is chosen to represent the failure envelope,

$$
q=3 A p^{\prime}+D
$$

where $A$ and $D$ are positive material constants. If the Drucker-Prager yield surface inscribes the Mohr-Coulomb yield surface, these constants can be related to a friction angle $\phi$ and a cohesion $\mathcal{C}$ by,

$$
3 A=\frac{2 \sin \phi}{\sqrt{3}(3-\sin \phi)}, \quad D=\frac{6 \mathcal{C} \cos \phi}{\sqrt{3}(3-\sin \phi)} .
$$

Figure 10 shows the stress path, along the dimensionless radius $r / r_{1}$, for two distinct boundary conditions at the wellbore: (left) permeable boundary and (right) semi-permeable boundary. The response of a dual porous medium $(2 \mathrm{P})$ with an average mass transfer coefficient $\eta^{*}=\eta_{a v}^{*}$ is compared with the associated single porous medium (A1P). The results are presented at $t=$ $6 \mathrm{~s}$ which corresponds to the maximum reduction in the effective radial stress (Figure 9).

The failure criterion line is calibrated to intersect the effective stress path representative of the dual porosity approach with a permeable condition on the wellbore. The values $A=$ 0.1 and $D=8 \mathrm{MPa}$ are chosen to describe the failure envelope, yielding a friction angle $\phi$ of $38^{\circ}$ and a cohesion $\mathcal{C}$ of $7 \mathrm{MPa}$.

The objective is to evaluate if the semi-permeable condition, modeled with the dual porosity approach and compared with the associated single porosity approach, affects the stability of the borehole positively or negatively.

Permeable boundary

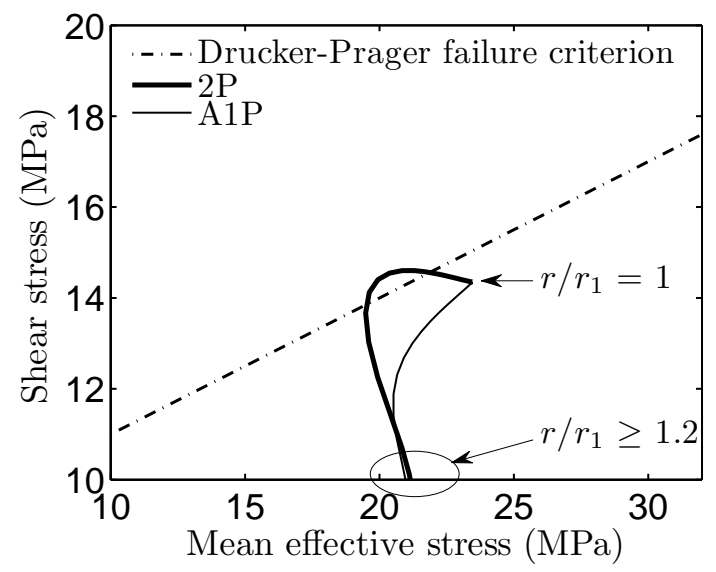

Semi-permeable boundary

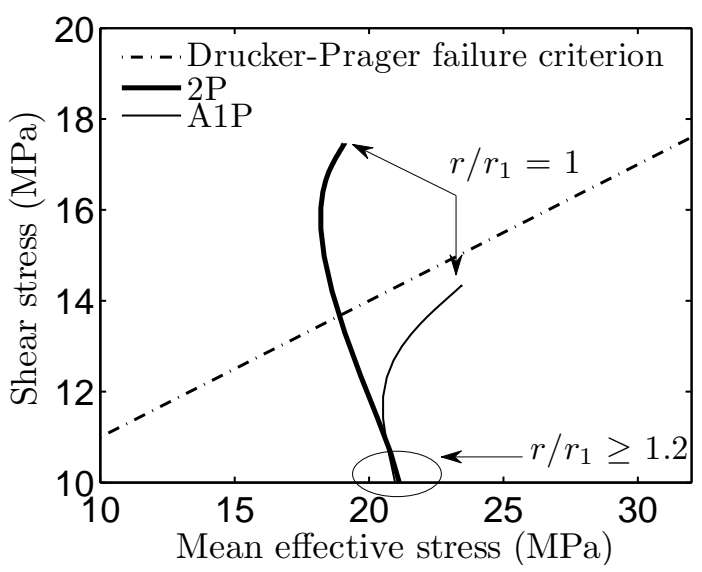

Figure 10: Effective stress path, in the mean effective stress $p^{\prime}$ - shear stress $q$ plane, along a radial direction $\mathrm{r} / \mathrm{r}_{1}$ for two distinct boundary conditions. The results are presented at time $6 \mathrm{~s}$ and for an average leakage parameter $\eta^{*}=\eta_{a v}^{*}$.

For a permeable boundary at the wellbore, the two stress paths at $r / r_{1}=1$ are located below the failure criterion. At a distance from the wellbore wall, the (A1P) effective stress path remains inside the failure envelope. In contrast to the $(\mathrm{A} 1 \mathrm{P})$ response, the $(2 \mathrm{P})$ effective stress path intersects the failure line and penetrates slightly into the failure domain. On the other hand, the $(2 \mathrm{P})$ stress path of the semi-permeable boundary condition is located outside the failure envelope for $r / r_{1} \leq 1.1$. As expected, the semi-permeable boundary induces a stronger collapse potential at the wellbore wall.

When accounting for a semi-permeable boundary condition, the effective radial stress is greatly reduced by the pore pressure in the vicinity of the wellbore (Figure 7) whereas the 
effective tangential stress remains essentially non affected. Consequently, the shear stress $q$ is significantly increased, while the mean effective stress is lower.

Figure 10 highlights that, in the dual porosity model, which contains few fissures and therefore uses a low leakage parameter coefficient, the potential for failure is greater than in the associated single porosity model. In addition, the semi-permeable boundary significantly increases the potential of failure of the borehole.

\section{Conclusion}

Diffusion mechanisms and mass transfer between materials with two porosities have been studied in the context of heavy-oil recovery rhrough thermal stimulation. A mechanical model for poroelastic dual porous media has been extended to account for thermal loading in a previous work [16]. The fully coupled thermo-hydro-mechanical system has been specified for mixtures that are in thermal equilibrium and for diffusion dominated media. The governing equations have been presented to characterize thermo-hydro-mechanical coupled behavior. A finite element approximation has been outlined and the nonlinear field equations integrated via an implicit time marching scheme and solved using a full Newton-Raphson procedure. This finite element analysis has been employed for a vertical borehole problem.

A parameter analysis has been developed to study the influence, in the vicinity of the wellbore, of the leakage parameter on the pressures of the pore and of the fissure network and on the effective stresses. The rise in the thermally induced pore pressure is more pronounced when the leakage parameter is low, representing a sparsely fissured media; whereas the response of the fissure network pressure is induced by the pore pressure dissipation and is therefore more pronounced for high leakage parameter. The effective stress is mostly controlled by the pore pressure, which induces a reduction in compressive effective stress for low leakage parameter values.

The highest reduction in the compressive effective stress occurs at a very short time after the loading, when the mass transfer has not resulted in the dissipation of excess pore pressure into the fissure network. It is also shown that dual porosity media display a higher failure potential compared with an associated single porosity medium. In addition, a semi-permeable boundary condition on the porous matrix greatly increases the failure potential compared with a permeable boundary condition at the wellbore lining.

Consequently, the single porosity approach underestimates the failure potential of fissured reservoirs. A consistent dual porosity approach is required for an accurate prediction of the potential for thermally induced wellbore failure in fractured porous media.

\section{Acknowledgments}

This work is supported by a PhD fellowship from the French Ministry of Higher Education. RG would like to thank the Association Française des Femmes Diplomées des Universités (AFFDU) and the Institut National Polytechnique de Grenoble for their travel grants. 


\section{Appendix A. Finite element sub-matrices}

The finite element sub-matrices of the weak formulation in eqns (19-22) are listed below:

- the four element matrices contributing to the balance of momentum of the mixture,

$$
\begin{array}{lll}
\mathbf{E}_{\mathbf{u u}}^{e}=\int_{V^{e}}\left(\mathbf{B}_{\mathbf{u}}\right)^{\mathrm{T}} \mathbf{D}_{\mathbf{e l}} \mathbf{B}_{\mathbf{u}} d V^{e}, & \mathbf{C}_{\mathbf{u p}}^{e}=\int_{V^{e}}\left(\nabla \mathbf{N}_{\mathbf{u}}\right)^{\mathrm{T}} \mathbf{N}_{\mathbf{p}} d V^{e} \\
\mathbf{C}_{\mathbf{p u}}^{e}=\int_{V^{e}}\left(\boldsymbol{\nabla} \mathbf{N}_{\mathbf{p}}\right)^{\mathrm{T}} \mathbf{N}_{\mathbf{u}} d V^{e}, & \mathbf{C}_{\mathbf{u T}}^{e}=\int_{V^{e}}\left(\nabla \mathbf{N}_{\mathbf{u}}\right)^{\mathrm{T}} \mathbf{N}_{\mathbf{T}} d V^{e}
\end{array}
$$

- the matrices describing the hydraulic conductivity and the thermal conductivity,

$$
\begin{aligned}
\mathbf{J}_{\mathbf{k}_{\mathbf{p}}}^{e} & =\int_{V^{e}}\left(\boldsymbol{\nabla} \mathbf{N}_{\mathbf{p}}\right)^{\mathrm{T}} \frac{k_{p}}{\mu_{p}} \nabla \mathbf{N}_{\mathbf{p}} d V^{e}, \quad \mathbf{J}_{\mathbf{k}_{\mathbf{f}}}^{e}=\int_{V^{e}}\left(\boldsymbol{\nabla} \mathbf{N}_{\mathbf{p}}\right)^{\mathrm{T}} \frac{k_{f}}{\mu_{f}} \nabla \mathbf{N}_{\mathbf{p}} d V^{e} \\
\mathbf{Q}_{\boldsymbol{\Lambda}}^{e} & =\int_{V^{e}}\left(\boldsymbol{\nabla} \mathbf{N}_{\mathbf{p}}\right)^{\mathrm{T}} \Lambda \nabla \mathbf{N}_{\mathbf{p}} d V^{e}
\end{aligned}
$$

- the three mass matrices,

$$
\begin{array}{ll}
\mathbf{M}_{\mathbf{p} \mathbf{p}}^{e}=\int_{V^{e}}\left(\mathbf{N}_{\mathbf{p}}\right)^{\mathrm{T}} \mathbf{N}_{\mathbf{p}} d V^{e}, & \mathbf{M}_{\mathbf{T} \mathbf{T}}^{e}=\int_{V^{e}}\left(\mathbf{N}_{\mathbf{T}}\right)^{\mathrm{T}} \mathbf{N}_{\mathbf{T}} d V^{e} \\
\mathbf{M}_{\mathbf{p} \mathbf{T}}^{e}=\int_{V^{e}}\left(\mathbf{N}_{\mathbf{p}}\right)^{\mathrm{T}} \mathbf{N}_{\mathbf{T}} d V^{e} &
\end{array}
$$

- the element force vectors,

$$
\begin{aligned}
& \mathbf{R}_{\mathbf{u}}^{e}=\int_{\partial V^{e}}\left(\mathbf{N}_{\mathbf{u}}\right)^{\mathrm{T}} \boldsymbol{\sigma} \cdot \hat{\mathbf{n}} d S^{e}+\int_{V^{e}}\left(\mathbf{N}_{\mathbf{u}}\right)^{\mathrm{T}} \mathbf{F} d V^{e} \\
& \mathbf{R}_{\mathbf{p}_{\mathbf{p}}}^{e}=\int_{\partial V^{e}}\left(\mathbf{N}_{\mathbf{p}}\right)^{\mathrm{T}} \mathbf{J}_{p} \cdot \hat{\mathbf{n}} d S^{e}+\int_{V^{e}}\left(\nabla \mathbf{N}_{\mathbf{p}}\right)^{\mathrm{T}} \frac{k_{p}}{\mu_{p}} \rho_{p} \mathbf{g} d V^{e} \\
& \mathbf{R}_{\mathbf{p}_{\mathbf{f}}}^{e}=\int_{\partial V^{e}}\left(\mathbf{N}_{\mathbf{p}}\right)^{\mathrm{T}} \mathbf{J}_{f} \cdot \hat{\mathbf{n}} d S^{e}+\int_{V^{e}}\left(\nabla \mathbf{N}_{\mathbf{p}}\right)^{\mathrm{T}} \frac{k_{f}}{\mu_{f}} \rho_{f} \mathbf{g} d V^{e} \\
& \mathbf{R}_{\mathbf{T}}^{e}=\int_{\partial V^{e}}\left(\mathbf{N}_{\mathbf{T}}\right)^{\mathrm{T}} \mathbf{q} \cdot \hat{\mathbf{n}} d S^{e}
\end{aligned}
$$

in which,

$$
\begin{aligned}
& \mathbf{N}_{\mathbf{u}}=\left[\begin{array}{cccccccc}
N^{1} & 0 & N^{2} & 0 & N^{3} & 0 & N^{4} & 0 \\
0 & N^{1} & 0 & N^{2} & 0 & N^{3} & 0 & N^{4}
\end{array}\right] \\
& \mathbf{N}_{\mathbf{p}}=\mathbf{N}_{\mathbf{T}}=\left[\begin{array}{lllll}
N^{1} & N^{2} & N^{3} & N^{4}
\end{array}\right],
\end{aligned}
$$

where $N^{1}, N^{2}, N^{3}$ and $N^{4}$ are the shape functions. $\mathbf{D}_{\mathbf{e l}}$ is the drained stiffness matrix,

$$
\mathbf{D}_{\mathrm{el}}=\left[\begin{array}{cccc}
\lambda+2 \mu & \lambda & 0 & \lambda \\
\lambda & \lambda+2 \mu & 0 & \lambda \\
0 & 0 & \mu & 0 \\
\lambda & \lambda & 0 & \lambda+2 \mu
\end{array}\right]
$$


and $\mathbf{B}_{\mathbf{u}}$ is the strain displacement matrix,

$$
\mathbf{B}_{\mathbf{u}}=\left[\begin{array}{cccccccc}
\frac{\partial N^{1}}{\partial r} & 0 & \frac{\partial N^{2}}{\partial r} & 0 & \frac{\partial N^{3}}{\partial r} & 0 & \frac{\partial N^{4}}{\partial r} & 0 \\
0 & \frac{\partial N^{1}}{\partial z} & 0 & \frac{\partial N^{2}}{\partial z} & 0 & \frac{\partial N^{3}}{\partial z} & 0 & \frac{\partial N^{4}}{\partial z} \\
\frac{\partial N^{1}}{\partial z} & \frac{\partial N^{1}}{\partial r} & \frac{\partial N^{2}}{\partial z} & \frac{\partial N^{2}}{\partial r} & \frac{\partial N^{3}}{\partial z} & \frac{\partial N^{3}}{\partial r} & \frac{\partial N^{4}}{\partial z} & \frac{\partial N^{4}}{\partial r} \\
\frac{N^{1}}{r} & 0 & \frac{N^{2}}{r} & 0 & \frac{N^{3}}{r} & 0 & \frac{N^{4}}{r} & 0
\end{array}\right]
$$

where $r$ and $z$ are the radial and the axial coordinates for the axi-symmetric case. Note that, in equations (A.5), the shape functions are the same for all primary variables.

\section{Appendix B. Definition of the matrices $K$ and $D$ in eqn (24)}

Identification of the sub-matrices of the stiffness matrix $\mathbb{K}$ and of the diffusion matrix $\mathbb{D}$, equation (24).

\begin{tabular}{llll}
\hline \hline $\mathbf{K}_{\mathbf{u u}}^{e}$ & $=\mathbf{E}_{\mathbf{u u}}^{e}$, & $\mathbf{K}_{\mathbf{u p} \mathbf{p}}^{e}=-\xi_{p} \mathbf{C}_{\mathbf{u p}}^{e}$ \\
$\mathbf{K}_{\mathbf{u p}}^{e}=-\xi_{f} \mathbf{C}_{\mathbf{u p}}^{e}$, & $\mathbf{K}_{\mathbf{u} \mathbf{T}}^{e}=-c_{T} / c \mathbf{C}_{\mathbf{u} \mathbf{T}}^{e}$ \\
$\mathbf{K}_{\mathbf{p}_{\mathbf{p}} \mathbf{p}_{\mathbf{p}}}^{e}=-\mathbf{J}_{\mathbf{k}_{\mathbf{p}}}^{e}-\eta \mathbf{M}_{\mathbf{p} \mathbf{p}}^{e}$, & $\mathbf{K}_{\mathbf{p}_{\mathbf{p}} \mathbf{p}_{\mathbf{f}}}^{e}=\eta \mathbf{M}_{\mathbf{p p}}^{e}$ \\
$\mathbf{K}_{\mathbf{p}_{\mathbf{f}} \mathbf{p}_{\mathbf{f}}}^{e}=-\mathbf{J}_{\mathbf{k}_{\mathbf{f}}}^{e}-\eta \mathbf{M}_{\mathbf{p} \mathbf{p}}^{e}$, & $\mathbf{K}_{\mathbf{p}_{\mathbf{f}} \mathbf{p}_{\mathbf{p}}}^{e}=\eta \mathbf{M}_{\mathbf{p p}}^{e}$ \\
$\mathbf{K}_{\mathbf{T} \mathbf{T}}^{e}=-\mathbf{Q}_{\mathbf{\Lambda}}^{e}$. & & \\
\hline \hline
\end{tabular}

and,

\begin{tabular}{llll}
\hline \hline $\mathbf{D}_{\mathbf{p}_{\mathbf{p}} \mathbf{u}}^{e}=-\xi_{p} \mathbf{C}_{\mathbf{p u}}^{e}$, & $\mathbf{D}_{\mathbf{p}_{\mathbf{p}} \mathbf{p}_{\mathbf{p}}}^{e}=-a_{p p} \mathbf{M}_{\mathbf{p} \mathbf{p}}^{e}$ \\
$\mathbf{D}_{\mathbf{p}_{\mathbf{p}} \mathbf{p} \mathbf{f}}^{e}=-a_{p f} \mathbf{M}_{\mathbf{p} \mathbf{p}}^{e}$, & $\mathbf{D}_{\mathbf{p}_{\mathbf{p}} \mathbf{T}}^{e}=-a_{p T} \mathbf{M}_{\mathbf{p} \mathbf{T}}^{e}$ \\
$\mathbf{D}_{\mathbf{p}_{\mathbf{f}} \mathbf{u}}^{e}=-\xi_{f} \mathbf{C}_{\mathbf{p u}}^{e}$, & $\mathbf{D}_{\mathbf{p}_{\mathbf{f}} \mathbf{p}_{\mathbf{p}}}^{e}=-a_{p f} \mathbf{M}_{\mathbf{v p p}}^{e}$ \\
$\mathbf{D}_{\mathbf{p}_{\mathbf{f}} \mathbf{p}_{\mathbf{f}}}^{e}=-a_{f f} \mathbf{M}_{\mathbf{p p}}^{e}$, & $\mathbf{D}_{\mathbf{p}_{\mathbf{f}} \mathbf{T}}^{e}=-a_{f T} \mathbf{M}_{\mathbf{p} \mathbf{T}}^{e}$ \\
$\mathbf{D}_{\mathbf{T} \mathbf{u}}^{e}=-c_{T} / c\left(\mathbf{C}_{\mathbf{u T}}^{e}\right)^{\mathrm{T}}$, & $\mathbf{D}_{\mathbf{T} \mathbf{p} \mathbf{p}}^{e}=-T^{0} a_{p T}\left(\mathbf{M}_{\mathbf{p} \mathbf{T}}^{e}\right)^{\mathrm{T}}$ \\
$\mathbf{D}_{\mathbf{T} \mathbf{p}_{\mathbf{f}}}^{e}=-T^{0} a_{f T}\left(\mathbf{M}_{\mathbf{p} \mathbf{T}}^{e}\right)^{\mathrm{T}}$, & $\mathbf{D}_{\mathbf{T} \mathbf{T}}^{e}=-a_{T T} \mathbf{M}_{\mathbf{T} \mathbf{T}}^{e}$ \\
\hline \hline
\end{tabular}

\section{References}

[1] L.L. Lake, R.L. Schmidt and P.B. Venuto, A niche for enhanced oil recovery in the 1990s, Schlumberger Oilfield Review: January, pp. 55-61, 1992.

[2] D.F. McTigue, Flow to a heated borehole in porous thermoelastic rock: analysis, Water Resources Research, 1990, 26(8), 1763-1774.

[3] D.F. McTigue, Thermoelastic Response of Fluid-saturated porous rock, Journal of Geophysical Research, 1986, 91, 9533-9542. 
[4] Y. Wang and E. Papamichos, Conductive heat flow and thermally induced fluid flow around a well bore in a poroelastic medium, Water Resources Research, 1994, 30(12), 3375-3384.

[5] X. Chen, C.P. Tan and C.M. Haberfield, Guidelines for efficient wellbore stability analysis, International Journal of Rock Mechanics \& Mining Sciences, 1997, 34, 3-4.

[6] G. Chen and R.T. Ewy, Thermoporoelastic effect on Wellbore stability, Society of Petroleum Engineers Journal, 2005, 10(2), 121-129.

[7] M.K. Rahman, D. Naseby and S.S. Rahman, Borehole collapse analysis incorporating timedependent pore pressure due to mud penetration in shales, Journal of Petroleum Science and Engineering, 2000, 28, 13-31.

[8] J. Zhang, M. Bai and J.C. Roegiers, Dual-porosity poroelastic analyses of wellbore stability, International Journal of Rock Mechanics \& Mining Sciences, 2003, 40, 473-483.

[9] G. Chen, M.E. Chenevert, M.M. Sharma and M. Yu, A study of wellbore stability in shales including poroelastic, chemical, and thermal effects, Journal of Petroleum Science and Engineering, 2003, 38, 167-176.

[10] Y. Abousleiman and S. Ekbote, Solutions for the Inclined Borehole in a Porothermoelastic Transversely Isotropic Medium, ASME Journal of Applied Mechanics, 2005, 72, 102-114.

[11] Y. Wang and M.B. Dusseault, A coupled conductive-convective thermo-poroelastic solution and implications for wellbore stability, Journal of Petroleum Science, 2003, 38, 187-198.

[12] W.K.S. Pao, R.W. Lewis and I. Masters, A fully coupled hydro-thermo-poro-mechanical model for black oil reservoir simulation, International Journal for Numerical and Analytical Methods in Geomechanics, 2001, 25, 1229-1256.

[13] M.A. Biot, General theory of three-dimensional consolidation, Journal of Applied Physics, 1941, 12, 155-164.

[14] G.I. Barenblatt, U.P. Zheltov and G.H. Kochina, Basic Concepts in the Theory of Seepage of Homogeneous Liquids in Fissured Rocks, Journal of Applied Mathematics and Mechanics, English Translation, 1960, 24, 1286-1303.

[15] I. Masters, W.K.S. Pao and R.W. Lewis, Coupling Temperature to a Double-porosity model of deformable porous media, International Journal for Numerical Methods in Engineering, 2000, 49, 421-438.

[16] N. Khalili and A.P.S. Selvadurai, A Fully Coupled Constitutive Model for Thermo-HydroMechanical Analysis in elastic media with double porosity, Geophysical Research Letters, 2003, 30(24), 7-1.

[17] R. Nair, Y. Abousleiman and M.M. Zaman, A Finite Element Porothermoelastic Model for dual-porosity media, International Journal for Numerical Methods in Engineering, 2004, $28,875-898$.

[18] R. Nair, Y. Abousleiman and M.M. Zaman, An application of the dual-porosity porothermoelastic approach in naturally fractured porous media, Poromechanics II, Auriault J.L. et al. eds, 2nd Biot Conference on Poromechanics, Grenoble, France, August 26-28, 2002, Swets \& Zeitlinger, 2002, 509-514. 
[19] N. Khalili and S. Valliappan, Unified Theory of Flow and Deformation in double porous media, European Journal of Mechanics - A/Solids, 1996, 15(2), 321-336.

[20] N. Khalili, Two-phase fluid flow through fractured porous media with deformable matrix, Water Resources Research, 2008, 44, 1-12.

[21] B. Loret and N. Khalili, Thermo-mechanical potentials for unsaturated soils, CISM Courses and Lectures no. 426 'Advanced Numerical Applications and Plasticity in Geomechanics', Udine, 2000, Edited by D.V. Griffiths and G. Gioda, Springer Wien New York, 253-276.

[22] N. Khalili and B. Loret, An elasto-plastic model for non-isothermal analysis of flow and deformation in unsaturated porous media: formulation, International Journal of Solids and Structures, 2001, 38(46-47), 8305-8330.

[23] B. Loret and N. Khalili, A three-phase model for unsaturated soils, International Journal for Numerical and Analytical Methods in Geomechanics, 2000, 24, 893-927.

[24] J.B. Warren and P.J. Root, The Behaviour of Naturally Fractured Reservoirs, Society of Petroleum Engineers Journal, 1963, 3, 245-255.

[25] N. Khalili, Coupling effects in double porosity media with deformable matrix, Geophysical Research Letters, 2003, 30(22), 2153.

[26] N. Khalili, S. Valliappan and C.F. Wan, Consolidation of Fissured Clays, Géotechnique, 1999, 49(1), 75-89.

[27] J. Bear and M.Y. Corapcioglu, A mathematical model for consolidation in a thermoelastic aquifer due to hot water injection or pumping, Water Resources Research, 1981, 17, 723736.

[28] T.J.R. Hughes, The Finite Element Method. Linear Static and Dynamic Finite Element Analysis, Prentice-Hall Inc., Englewood Cliffs, New Jersey, 1987.

[29] R.K. Wilson and E.C. Aifantis, On the Theory of consolidation with double porosity, International Journal of Engineering Science, 1982, 20(9), 1009-1035.

[30] J. Kestin, A Course in Thermodynamics, Hemisphere Pub, 1979, Waltham, Massachussets: Blaisdell Pub. Co, Volume 2.

[31] D.C. Drucker and W. Prager, Soil mechanics and plastic analysis for limit design, Quarterly of Applied Mathematics, 1952, 10(2), 157-165.

[32] R.W. Zimmerman, T. Hadgu and G.S. Bodvarsson, Development of a dual-porosity model for vapor-dominated fractured geothermal reservoirs using a semi-analytical fracture/matrix interaction term, Proceedings, Eighteenth Workshop on Geothermal Reservoir Engineering, Stanford University, Stanford, California, January 26-28 1993.

[33] M. Lu and L.D. Connell, A dual-porosity model for gas reservoir flow incorporating adsorption behaviour-part I. Theoretical development and asymptotic analyses, Transport in porous media, 2007, 68(2), 153-173. 